\title{
Tactful Networking: Humans in the communication loop
}

\author{
Rafael Lima Costa, Aline Carneiro Viana, Artur Ziviani, and Leobino Nascimento Sampaio \\ COPYRIGHT NOTICE \\ This is the accepted version (not the IEEE-published version) of the paper 10.1109/TETCI.2020.3039520 \\ The Copyright belongs to IEEE Transactions on Emerging Topics in Computational Intelligence (Volume: 5, Issue: 1, Feb. 2021)
}

\begin{abstract}
This survey discusses the human-perspective into networking through the Tactful Networking paradigm, whose goal is to add perceptive senses to the network by assigning it with human-like capabilities of observation, interpretation, and reaction to daily-life features and associated entities. To achieve this, knowledge extracted from inherent human behavior in terms of routines, personality, interactions, and others is leveraged, empowering the learning and prediction of user needs to improve QoE and system performance while respecting privacy and fostering new applications and services. Tactful Networking groups solutions from literature and innovative interdisciplinary human aspects studied in other areas. The paradigm is motivated by mobile devices' pervasiveness and increasing presence as a sensor in our daily social activities. With the human element in the foreground, it is essential: (i) to center big data analytics around individuals; (ii) to create suitable incentive mechanisms for user participation; (iii) to design and evaluate both humanaware and system-aware networking solutions; and (iv) to apply prior and innovative techniques to deal with human-behavior sensing and learning. This survey reviews the human aspect in networking solutions through over a decade, followed by discussing the tactful networking impact through literature in behavior analysis and representative examples. This paper also discusses a framework comprising data management, analytics, and privacy for enhancing human raw-data to assist Tactful Networking solutions. Finally, challenges and opportunities for future research are presented.
\end{abstract}

Index Terms-Human behavioral perception, human-aware, next-generation networks.

\section{INTRODUCTION}

Computing and networking systems design are increasingly dealing with user expectations. The Multi-Protocol Label Switching (MPLS) and Software-Defined Networking (SDN) are examples of technologies apt to assist operators in providing QoS and QoE services through user-oriented traffic differentiation. In the last decade, several initiatives based on direct user involvement gained attention as enablers for wireless communications, e.g., User-centric Networks, Deviceto-Device Communication (D2D), and recently, User-in-theloop and Human-in-the-loop proposals. Although having the user as the central concern, the first solutions in User-centric Networks still did not see the user as an individual, but rather as a network active element. Hence, frequently, only

Rafael L. Costa is with École Polytechnique/IPP and Inria, France and with Federal University of Bahia, Brazil (e-mail: rlimacosta@ufba.br); Aline C. Viana is with Inria, Palaiseau, France (e-mail: aline.viana@inria.fr); Artur Ziviani is with the National Laboratory for Scientific Computing (LNCC), Brazil (e-mail: ziviani@ Incc.br); and Leobino N. Sampaio is with Federal University of Bahia, Brazil (e-mail: leobino@ufba.br). This work was supported in part by the FAPERJ, FAPESB, CNPq, CAPES, INCT-CiD, EMBRACE associate team Inria project, and ANR MITIK project (PRC AAPG2019). user features measured from the network point of view were considered, ignoring the intrinsic ones from human activity, e.g., daily routines and personal preferences.

In the D2D initiatives, considered one of the $5 \mathrm{G}$ enablers [1], different proposals started to focus on human aspects, such as mobility and social links. Lately, "User-inthe-loop" and "Human-in-the-loop" research brought more attention to taking advantage of human behavior's unique features to leverage computing powers owned by users. In [2], authors survey recent "Human-in-the-loop" efforts and discuss topics such as crowdsensing applications and data assembly, incentive mechanisms for human-collaboration, human-data privacy, and exploring human factors through human-system interaction, user demand prediction, and learning-aided dynamic system control. In this survey, differently from previous research, we bring a human perspective closer to individual aspects, considering attributes that could link to performance, plus decoupling the human factor from the networking system point of view, targeting a more personalized network service. Therefore, our goal is a more in-depth investigation about which aspects of human behavior and their peculiarities could help the modeling of future mobile networks, including knowledge from other areas like psychology and philosophy.

This paper argues that the following paradigm shift is required: from a network controlling, tracking, and monitoring networking users to a network perceiving the needs and adapting to inherent behaviors of humans behind networking devices while respecting their privacy. We denominate this paradigm Tactful Networking and it calls for having, progressively, computer networks and mobile devices that understand and react to human-behavior characteristics. Tactful Networking groups concepts from human behavior research, not only from computer networking but also from other areas. One of the goals of this paper is to bring a comprehensive survey digging into the human-traits peculiarities, showing the trend in human-aware networking. Thus, this paper advocates that by considering the tactful networking paradigm, consolidated technologies (e.g., MPLS, SDN) and novel ones can help achieve better QoE-aware services over the Internet.

Future wireless network generations, including their models, architectures, protocols, communication types, and related technologies, are expected to be tactful, i.e., sensitive and adaptive to human context, behavior, and interest. The tactful networking goal is to offer services that consider daily inherent human characteristics and behavior, including capabilities and perception, to fulfill user expectations more naturally.

The study of human behavior has occurred for years in psychology, physics, and sociology. Recent advances in big data 
collection/analysis, machine learning, edge computing, haptic systems, human-computer interaction, computational social science, and other directions have shown that human-behavior investigation is also vital in networking [3], [4]. Examples of individual characteristics include mobility, preferences, interests, humor, sharing wills, age, socioeconomic status, or contextual routines, which can be studied to offer a proper human-aware network service. Incorporating the capability of human behavioral perception into networking solutions has the potential to bring an open-ended vision for what networks should be able to do while fostering the deployment of new $5 \mathrm{G}$ applications. This paper surveys literature works in this direction. The context herein discussed strengthens the need for the design of incentive and recommendation mechanisms and the emergence of innovative networking applications and services. It also offers support to new business models that, overall, are getting increasing interests from carriers and application developers. The implementation of tactful networking can become a win-win situation. The user will have better QoE when accessing an operator network closer to her requirements (thus bringing her the feeling of accessing personally designed services; one of the future considerations envisaged in 6G). Meanwhile, an operator can maximize profit by managing resources and system performance with lower cost while offering new user-behavior-based services.

This paper's central focus is thus to discuss why and how the humans can be under the spotlight for future generation network architectures. This paper's contributions feature a survey on human behavior's evolution as a central element for dealing with challenges into networking solutions. We cover over a decade of initiatives through a timeline in which we see a considerable change towards understanding the human role in computer networks. This change culminates into what we call the Tactful Networking paradigm. Furthermore, this survey discusses the Tactful Networking paradigm, which groups several human aspects applied in prior solutions, and other ones from different areas of knowledge, such as psychology and philosophy. This paper investigates more indepth into the particularities of these human-traits through surveying literature works on behavior analysis. Essential findings in each human trait, including challenges for their use, examples of services, and applications, are also featured. Moreover, this paper debates over a general framework for processing human-behavior raw data and preserving its privacy while assisting Tactful Networking applications. This framework comprises examples of data sources, challenges, and solutions in the context, serving as a best-practice guide for dealing with raw human data into networking. Finally, the paper highlights deliberations about what is necessary for Tactful Networking solutions to evolve, pointing out future directions for further investigation, and how users and enterprises can benefit from the discussed paradigm. The remainder of the paper is structured as follows. In Section II, we survey the evolution from user-centric to human-aware communications. Section III presents the Tactful Networking perspective, bringing innovative discussions from the human point-of-view in computer networks. Section IV features a framework discussion with practices on data management, analytics, and privacy, employed to extract useful knowledge from different heterogeneous data sources of human behavior. Finally, Section V concludes the survey, also presenting the research challenges in the area.

\section{FROM USER-CENTRIC TO HUMAN-AWARE COMMUNICATIONS}

Literature solutions based on a user-centric design brought more attention not only to user requirements, expectations, and QoE but also on how the user participation can help to solve challenges in the context of mobile networks. These useroriented approaches assisted mobile network design in different challenges, including energy and spectrum efficiency, routing, computing capacity, and capillarity extension. This section surveys the human-perspective into networking through a timeline, depicted in Figure 1. This timeline summarizes the research evolution from user-centric communication to more recent human-aware approaches. We highlight essential works, directions, milestones, and accomplishments for over a decade, showing a considerable change towards understanding the human role in computer networks. As the survey covers an extended period, more examples and citations are from more recent research. The Tactful Networking perspective (Section III) features different human-traits applied in some of these previous works herein mentioned, added to other insights from interdisciplinary research featured on this survey.

From 2008, we highlight the "user as provider" concept from user-centric communication [5], a solution for capillarity extension and data offloading through user participation. The user-centric main idea was for the user to act as a service/connectivity provider in specific scenarios: network edge, crowded places, and disasters. Despite many challenges identified [5], most discussions were still from a network, device, performance, and services perspectives, such as routing, access control, power consumption, and data privacy.

In 2009, the user mobility context [6] was a key topic with discussions about the main mobility aspects, the limitation of current protocols for user-centric models, and a user-centric future internet vision. The impact of user-location sensing [7] revealed issues related to control and privacy. It also appeared research [8] about economic interactions between users, ISPs, and community providers to build incentive mechanisms guided by user characteristics such as uncertainty, selfishness, and sensitivity to device resources consumption [2].

During 2010, the importance of smart devices and online social networks (OSNs) sensing appeared in [9]. They gather user attributes and social graphs from social networks to infer other users' characteristics. This kind of sensing involves collecting mobile datasets from user devices to assist opportunistic routing architectures, such as [10]. They analyze and classify users' interactions according to their interests and activity. Further, human mobility analysis kept appearing. In [11], user contacts are mapped to social graphs for achieving better DTN routing performance. Studies in user behavior, preferences, attributes, and context started to be more common. Userprovided parameters served as input to advanced network provisioning [12]. Approaches for user-centric 4G appeared, 


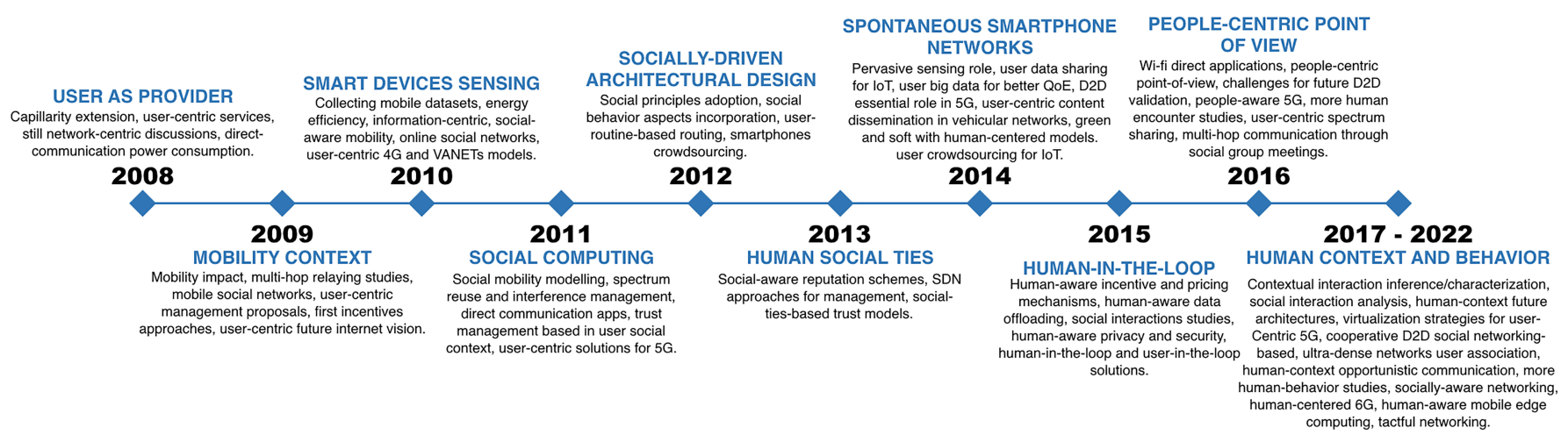

Fig. 1. Timeline with the evolution of user-centric initiatives to human-centered future network generations.

like the QoS-based network selection algorithm relying on user preferences in [13]. Furthermore, in [14], cooperative usercentric communication and network selection to achieve user satisfaction while overcoming network conflicting interests.

From 2011 we highlight social computing studies in different initiatives, such as human social aspects in mobility modeling for improving mobile network operation [15], access control, trust management [16], and privacy [17]. Among the characteristics considered were similarity of user-profiles, reputation, and friendship history in social networks. Userprovided networks/services relying on user-preferences targeted energy efficiency, QoS [18], among others. Mobility analysis appears in different domains, such as investigating mobility models, network selection, and handover techniques based on displacement prediction, context, user profiles, and preferences [19]. Task composition in urban computing based on social, spatial, and temporal aspects also appeared [20]. In [21], authors affirm future internet will be user-centric and context-aware. In [22], a novel user-centric architecture for $5 \mathrm{G}$, gives the mobile terminals the power to change the Radio Access Technology based on user criteria.

Discussions about a socially-driven Internet architectural design were frequent in 2012. In [23], the role of prospective consumers (also services providers) appears as an Internet trend of user empowerment. Social aspects appeared in multiple initiatives. Among those, data forwarding and privacy, social similarities from mobility, opportunistic routing based on social structures from daily routines, a user-centric socialnetwork framework, social user-profiling, social context-based routing, user behavior in "smart-grid" communications, and user cooperation in peer-to-peer systems [24] and in energyefficient routing. A QoS-based incentive mechanism for usercentric networks appears in [25]. User-centric resource allocation, cache distribution, energy-efficiency [26], service exposure model [27], connection sharing, and user sensing for wireless access are other examples. In mobility management, aspects studied include user-device resources, locations, user-profile, context, preferences, user-perspective mobility schemes, identity, and handoff/handover solutions. A survey on handover management in mobility architectures is in [28].

In 2013, human social ties studies appeared frequently. Challenges and solutions from OppNets and DTN to Mobile
Social Networks (MSNs) are in [29], focusing on works related to security, privacy, and trust in MSNs. Environment, context, reputation, community check, and other aspects appeared in trust management, cooperative models, and mobility analysis [30]. A survey [31] on mobile social networking middleware brought a valuable remark about opportunistically created communities that should be determined not only by shared interests or contacts but also by mobility-related context like physical location and co-presence. The paper [32] calls attention to user empowerment discussions in data offloading, where the decision making shall not belong only to the network, but to the user. A debate about a self-adaptive system for data dissemination in opportunistic networks relying on a recognition heuristic with human-brain-like decisions is in [33]. Social ties and user characteristics, such as communities, visited locations, friendship, selfishness, people's daily routines, and others, were used in MSNs, DTN, and routing initiatives [34], [35]. In [36], privacy, and anonymity of user sensitive data were a concern. In [37] is discussed the importance of users as participatory sensors to understand city dynamics and their inhabitants' urban behavioral patterns. In [38], user-perceivable metrics such as application quality, energy, and monetary costs were applied to optimize network use. User ubiquity applied to extend network service, interactivity, and interoperability in rural areas and opportunistically share content based on user preferences. Human social-ties applied to satisfy user requirements in bandwidth allocation.

Meanwhile, in 2014, the potential of spontaneous smartphone networks and cooperative relaying was considered in different works, making services adaptive to both user and network requirements [39], [40]. Discussions in Device-todevice communications for $5 \mathrm{G}$ included security, privacy, interference management, resource allocation, and pricing models [41]. Moreover, in the 5G context, we found works about reaching a superior user experience alongside new services and applications [42], green and soft future network rethinking the cell-centric design [43], and a consumer-oriented 5G design. Other works proposed user-centered routing with user requests as central requirements for achieving optimal communication performance, and social-aware routing in user-centric networks [44]. In [45], a resource allocation mechanism relies on a user-centric network utility maximization problem with user 
traffic-profile status as input. In [46], user-centric solutions for communication services and network management focus on the tradeoff between security and performance. A Survey on Virtual Sensor Networks in terms of security and privacy is in [47]. These networks monitor large groups of people and store sensitive individuals' information, such as personal identity, behavior, interaction, preferences, and mobility. In [48], vehicular social networks are discussed through a taxonomy for content-dissemination approaches and a framework to support user satisfaction. Incentive and reputation mechanisms appeared in user-provided networks [49], IoT, and social networks. QoE and QoS are discussed in [50], [51]. Among the aspects considered were moving content closer to the edge to improve network quality, content-adaptation from user preferences, user-participation schemes to indicate satisfaction, and user recommendations. User-Centric Network Management was also a relevant topic [52] linked with technologies such as SDN to mitigate the problem of sharing limited network capacity and resources efficiently and fairly. Context information, mobility management [53] in opportunistic networks, and distributed social information applied to different initiatives. The lack of testbeds and datasets for mobility evaluation motivated research while ad hoc and mesh networks surveys [54], [55] pointed out future people-centric networking. The term "User-in-the-loop" appeared in [56] in a system where the user can actively participate in networking congestion situations.

From 2015 to 2017, human-aspects started to be even more frequent in cooperative networking. During 2015, we highlight Human-in-the-loop and User-in-the-loop approaches [57], [58], considering aspects such as human intents, psychological states, emotions, and actions inferred through sensory data. User-centric and user-provided wireless networking proposals are in [59], [60]. They emphasize people-centric networking, user interests, and social connections in different scenarios. In [61], appears an SDN-based 5G architecture relying on user location information. A survey on data offloading techniques, including those based on user cooperation, geographical context, and content popularity, is in [62]. A user-centric model for virtualized security at the network edge relying on user profiles appears in [63]. Another frequent subject was mobile users crowdsensing systems [64]. This subject appeared in different areas such as user privacy, reputation mechanisms, and considering aspects as user social preferences, traffic profile, environment data, and user behaviors (in terms of honesty). Mobility and Online Social Networks [65] studies kept appearing in opportunistic routing, forwarding, and dissemination, handover management, recommendation systems (based on user opinions, preferences, and behavior in OSN), among others. User satisfaction, QoE, and QoS are in [66], reinforcing the user protagonism in networking services.

In 2016, terms like people-centric [67] kept appearing, reinforcing the importance of understanding the human behind a device - an individual sharing contents, experiences, and acting as a mobile virtual sensor. In the $5 \mathrm{G}$ context, user-centric solutions, smooth user experience, and sensing user information to allocate resources appeared [68]. Research in Ultra-Dense Networks [69] also breaks the network's paradigm controlling its users to an architecture closer to their requirements. In [1], are pointed out technological breakthroughs that would bring a renaissance to wireless communication networks, including D2D communication, network ultra-densification, and big data analytics (due to the amount of data generated by the mobile users). Data Offloading appeared relying on user-device context (e.g., energy constraints), QoE, mobility, and other aspects. Advances in mobility and handover management, based on user preferences, QoE, power status, movements, localization techniques, and geo-analysis, were also found [70]. Works with mobile crowdsourcing, extracting intelligence from OSNs, contextual information, social relationships, privacy [71], security, QoS, reputation, and incentive mechanisms appeared. The importance of micro properties (known as EGO Networks) from personal networks of users in OSN is in [72]. They show that the structural properties of OSNs are similar to social networks formed offline. Understanding these properties can be essential to the creation of services for the future internet. User data aggregation in OSNs, the study of social relationships between people, and context applied to encounters coordination. In [73], a user-centric QoE prediction algorithm with machine learning, was proposed. A DTN Routing survey is in [74] and relates to Information-Centric Networks, IoT, and other architectures that can benefit from human-behavior information. User-centric versus Networkcentric resource management appears in [75]. Other works included scheduling based on user cooperation, user-centric scheduling for flexible 5G design [76], an opportunistic data dissemination strategy in D2D, opportunistic D2D routing relying on social group meetings [77], user-centric energyefficient wireless energy transfer, and opportunistic data transmission of urban sensing applications [78].

From 2017, we highlight human-in-the-loop and user-in-theloop works, reinforcing the need for learning, adapting, and steering user behavior to exploit the human factor in future ubiquitous mobile systems [2], [79]. Other works included user preferences applied into several solutions: home networks management; municipal Wi-Fi deployment based on usage patterns and who are the users; group communication schemes in opportunistic ad-hoc networks; device-to-device communication dealing with user need to have more efficient utilization of network resources including energy [80]; ultra-dense networks [81], mobile edge networks [82], extending user-centric Internet services with peer-to-peer interactions, and contextaware resource allocation. A user-centric context-aware radio access technology selection for $5 \mathrm{G}$ is in [83]. The paper [84] discusses $5 \mathrm{G}$ trials, challenges, and deployment. Authors argue that D2D, M2M, V2V, and IoT will play an important role in $5 \mathrm{G}$. Here in this section, we cited different works applying user characteristics to solve challenges in the context of these communication types. Other works included user-provided networks with incentive mechanisms [85], cooperation-based cache [86], extracting social relations from users' ratings [87], and mobility behavior analysis. Online Social Networks research kept appearing linked to cooperative D2D based on social aspects [88], decentralized OSNs [89], and social network analysis methods in behavioral information security. According to [90], we are moving towards the $5 \mathrm{G}$ era, witnessing a 
transformation in the way networks are designed and behave, with the end-user placed at the epicenter of any decision.

In 2018, the trend related to user-centric initiatives, userin-the-loop, and human-in-the-loop continued. In [91], spatial and social awareness are combined to outperform state-ofart D2D opportunistic routing protocols. In [92], a survey on IoT future proposes a four-layer architecture, including a sensing layer. A "human-in-the-loop" 5G system in [93] combines prediction from big data analytics centered in user demand with pushing and caching. In [94], a proposal for data offloading relies on user participation. They affirm most previous works in this context ignored user device constraints (such as battery power and computing capabilities) while their solution considers these parameters. In [95], user reliability propagation relies on mobile social network interactions. Their solution detects malicious and selfish nodes that affect network efficiency. An information-centric caching scheme through D2D in 5G is in [96]. Other examples are user-centric D2D content-sharing [97], human-in-the-loop radio resource allocation for haptic communications [98], user-centric ultradense-networks [99] dealing with resource allocation, content popularity learning from user spatiotemporal mobility, usercentric cooperative caching based on network topology, traffic distribution, channel quality, and file popularity [100], and dynamic AP grouping based on user mobility and behavior.

Finally, in 2019, the human-user context is linked with subjects such as urban computing, machine learning, distributed spectrum sharing in dynamic networks, wireless virtualization, and handover. Many of these initiatives link to future 5G. In [101] appears a survey on location-based social networks (LBSNs) as a source of user data to leverage urban computing solutions. A machine learning solution linked with $5 \mathrm{G}$ usercentric ultra-dense networks (UUDN) capable of improving network performance appears in [102]. A novel user-centric networking model where each user, based on uncertainty and their traffic model, can serve as access points for other users in their vicinity is in [103]. In [104], a 5G user-centric wireless access virtualization proposal allows users to benefit from a set of transmission points selected according to their environment and QoS requirements. This architecture represents a rupture to the traditional cell-centric scheme. In [105], handover opportunities in user-centric networks rely on user characteristics such as direction and speed.

In the upcoming years, future generation mobile networks will deal with human context, behavior, and information, considering aspects such as mobility, interactions, social ties, traffic profile, personality, and others discussed in the following section. Therefore, based on the discussions above and throughout the years, network models and solutions are becoming linked with a new level of understanding where human characteristics will have to be considered to offer better and more personalized service through tactful networking.

\section{THE TACTFUL NETWORKING PERSPECTIVE}

Traditionally, the design of computer networks happened through a service-provider perspective. This results in a gap between the way networking protocols and services are created (e.g., usually limited to service providers' needs or types of application) and the everyday user behavior or needs. We observe this gap in techniques currently used to optimize network performance. In particular, many of them adapt to network conditions (e.g., physical link conditions, topology changes) and are protocol or service-specific (e.g., successful delivery of messages or geographical network coverage).

New expected mobile applications and requirements raised by $5 \mathrm{G} / 6 \mathrm{G}$ that rely on accurate users' behavior or locations would worsen such a gap. All this stresses the urgency for a proactive accommodation of human behavior, which refers to the anticipation of users' behaviors, allowing the services and the communication systems to adapt to it proactively [106], [107]. As an example of human behavior accommodation, consider mobility behavior and 5G. Although the concept of Mobility Management as a Service (MMaaS) is introduced in $5 \mathrm{G}$, existing implementations are still feedback or signalingrelated. Mobility-triggered decisions are based on devices signaling, which incur extra load. Furthermore, most of the in-discussion MMaaS specifications are architecture, protocol, or radio-related (e.g., SDN, optimizations on transport or mac layer protocols, millimeter-wave communication). The mentioned specifications fail in provisioning high reliability, currently tackled through flow or connectivity redundancy. Finally, 5G is expected to manage "on-demand mobility": true AI is absent in 5G [107]. Hence, online mobility learning/training and in-advance inference of accurate future movements or individuals' mobility preferences (to novelty and diversity) constitute essential missed points in $5 \mathrm{G}$.

In an Internet that has become essentially mobile [108], it has become urgent for networking services to accommodate users' dynamic behaviors, no matter how dynamic they are or how uncertain their movements are meant to be. Behavior accommodation refers to the anticipation of users' behavior (e.g., movements, interests, etc.), allowing the services and communication systems to be proactively adaptive.

In particular, since humans nowadays are often carrying and interacting through smart-devices, most of our activities reflect our real lives onto the digital binary world. Thanks to smart-devices massive adoption, mobile applications create a digital footprint that directly reflects our routines, interest, and whereabouts. Hence, our behavior and individual characteristics directly impact how we demand network resources and what kind of resources are requested. In this context, large datasets are collected by various stakeholders to leverage digital footprints and better learn our tastes, habits, and social lives. All this pops up new opportunities to enforce the understanding of people's behaviors and leverage this understanding in networking solutions' design.

As a result, we discuss the need for future network design to take human behavioral aspects into account to optimize network resources, services, and performance. In this section, we review the Tactful Networking paradigm, the human behavior aspects that can be extracted and leveraged from digital footprints, and literature on behavior analysis. Some of these human-behavior aspects cited herein appear in solutions described in the previous section. Furthermore, this section deliberates about each human-traits' specific characteristics, also bringing other ones from interdisciplinary research. 


\section{A. The tactful networking concept}

The Tactful Networking research paradigm herein discussed is a reference to the objective of adding perceptive senses to the network by assigning it with the human-like capabilities of observation, interpretation, and reaction to daily life features and involved entities. It is valuable to mention that the Tactful Networking concept is not the same as Tactile Internet [109], [110]. The latter regards research initiatives in which network connectivity aims to deliver real-time physical tactile experiences remotely. Conversely, being tactful is having or showing skill and sensitivity in handling with people. It also aggregates diplomacy, perception, tact, and care. Therefore, a tactful network can be more precisely defined as a network that considers human behavioral characteristics (i) to foresee user needs and actions; (ii) to self-adapt to the inherent heterogeneity and uncertainty of individuals; (iii) while offering a better quality of experience and improving system efficiency.

Figure 2 illustrates a tactful networking ecosystem where the human element is the epicenter of future networking solutions. His surroundings feature different aspects of his behavior that can bring valuable information to the networking domain. Mobility patterns and interactions are examples applied (section II). Further, in this section, we discuss more aspects that can bring interesting insights into networking solutions. Among those, socio-demographic traits, socio-economic traits, and personality traits. More externally in the figure, we have several (but not limited to the ones herein listed) computer networking technologies or paradigms that already benefited or can benefit from the human-behavior data. Besides these and other technologies, new public-focused services (e.g., customized advertisement delivery or recommendations) or business models and opportunities can appear.

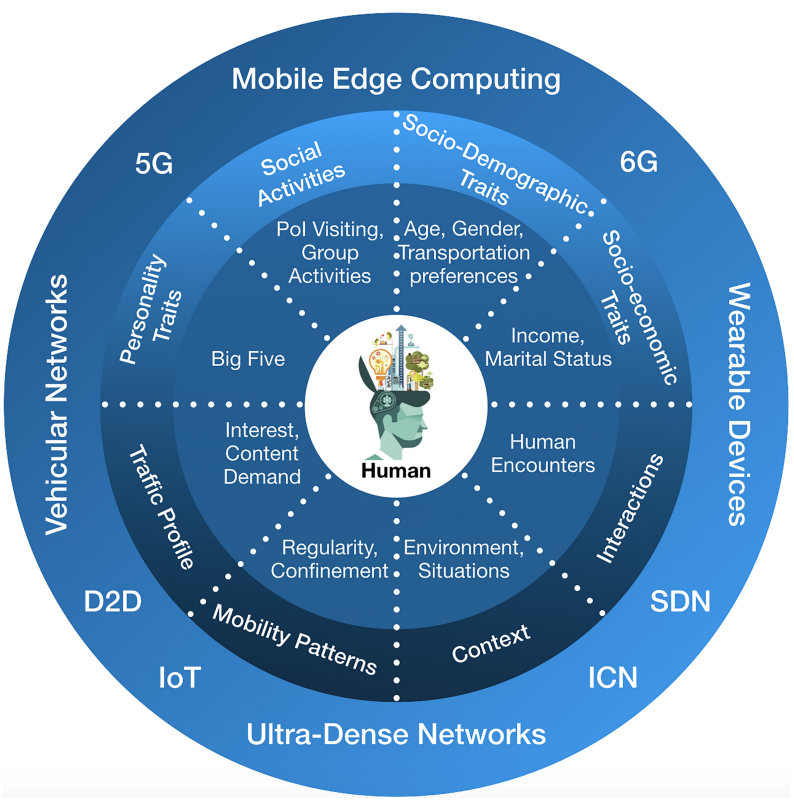

Fig. 2. Tactful Networking Ecosystem where human-related aspects are future mobile-networks enablers.

Table III-A summarizes some human multi-dimensional aspects to be considered. Following section features ideas behind their usability in tactful networking.

\section{B. Behavior analysis in the literature}

What tactful networking claims to consider are behaviors shaped by the fact that we are human beings. Some inherent examples are: (i) people habitually act as semi-rational entities, routinely moving and interacting within a reduced and predictable geographic landscape [113] and tending to meet the same people; (ii) people build their life-routine in particular areas that can also link to their personality and social ties; (iii) human decision-making process deals with entropy and uncertainty, led by situations such as conflict, paradox, and noise [127]. Hereafter, we discuss literature works entitled to capture and analyze human behaviors.

a) Mobility: Understanding human mobility has many applications in several areas, such as the spread of diseases, city planning, traffic engineering, targeted product advertisements, and networking resource allocation. As routines and habits dictate our life, mobility data is relevant for inferring behavior. For instance, in modern urban planning, understanding human travel patterns on the city level is essential. Similarly, mobile operators could better adapt their resource allocation or service provision if they understand their users' displacement tendencies. Further, over 24\% of Android applications build their services on top of human-mobility data. All this shows the importance of deciphering human motion.

Literature works have unraveled interesting properties of underlying large-scale mobility patterns: Recurrence and temporal periodicity of visited locations [113], [115]; Confinement (a small area an individual visits) [113], [115]; High predictability of human mobility [117]; Few unique network motifs (i.e., about 17) explaining the majority of daily human mobility [128]; Population trip distance and radius of gyration distribution following a power law [115]; A very high uniqueness of individual trajectories (i.e., four random time-stamped locations identify one user among 1.5 million individuals in $95 \%$ of the time) [129]; Few trips to new places outside an individual radius and about $25 \%$ of human mobility relating to new places visits [130]; Tendency to minimize their efforts (i.e., following the shortest path while moving). This phenomenon repeats independently of countries, cultures, or transportation means being used.

b) Personality Traits: In recent years, personality prediction has attracted interest from the computer science research community. Technologies, services, or applications can be improved to answer users' expectations and needs if such interested users' personality is known and better understood. For example, recommendations on new places to visit and novel experiences to seek could reach to individuals more disposed to enjoy the information. Alternatively, online social networks or crowdsensing applications could better suggest new activities or connect individuals with similar personalities and interests. For capturing individuals' personalities, the research community has been considering the Big 5 personality model [119]. It delineates the OCEAN traits, as follows: Openness (to experiences) $(O)$ is associated with intelligence, originality, creativity, and intellectual curiosity. Conscientiousness $(C)$ describes self-control, planning, and organizational skills. Extraversion $(E)$ accounts for assertiveness, positive 
TABLE I

KEY HUMAN ASPECTS FOR TACTFUL NETWORKING

\begin{tabular}{|c|c|c|}
\hline Human Aspect & Description & Services or Applications \\
\hline Context & $\begin{array}{l}\text { Relates to the logical or physical context in which an individual inter- } \\
\text { acts. Aspects such as time of the day, weather conditions, location-based } \\
\text { events [111], preferences (e.g., device interfaces, geographical areas [112], } \\
\text { tools, applications), among others, are considered. }\end{array}$ & $\begin{array}{l}\text { Recommendation or customized advertisement services; context-aware predic- } \\
\text { tion systems (the use of context allows decreasing the required visiting history } \\
\text { of users and improving accuracy prediction). }\end{array}$ \\
\hline Interactions & $\begin{array}{l}\text { Study the features underlying human physical encounters, such as regular- } \\
\text { ity [113], similarity [10], contact, or inter-contact duration in the temporal } \\
\text { graph of encounters among individuals. }\end{array}$ & $\begin{array}{l}\text { Interaction-based data offloading [62]; opportunistic applications or ser- } \\
\text { vices [53]; proximity-based social networks [89]; prevention of cybersecurity } \\
\text { attacks (e.g., understanding a malware propagation in mobile wireless networks) } \\
\text { as well as of epidemic disease propagation. }\end{array}$ \\
\hline Mobility & $\begin{array}{l}\text { Accounts for regularity [114], entropy, confinement [115], as well as time } \\
\text { periodicity [116] of visits. Location similarity [117], displacement profile, } \\
\text { important location and routine inference are also related. }\end{array}$ & $\begin{array}{l}\text { Network resource allocation [52] and optimization; content pre-fetching; urban } \\
\text { planning [101]; traffic engineering [118]; prevention of cybersecurity attacks } \\
\text { (e.g., understanding a malware propagation in mobile wireless networks) as } \\
\text { well as of epidemic disease propagation; crime prediction. }\end{array}$ \\
\hline $\begin{array}{l}\text { Personality } \\
\text { Traits }\end{array}$ & $\begin{array}{l}\text { Accounts for the OCEAN traits extracted through the Big5 model [119]: } \\
\text { Openness, Conscientiousness, Extraversion, Agreeableness, Neuroticism. } \\
\text { Relates to the understanding of the influence that personality traits may } \\
\text { have on individuals' behaviors (e.g., motion [120], physical/social relation- } \\
\text { ships [121], preferences) or context. }\end{array}$ & $\begin{array}{l}\text { Recommendation [122], [123] or customised advertisement services; incentive- } \\
\text { based approaches; message propagation; smartphone usage understand- } \\
\text { ing [124]; trust-management models. }\end{array}$ \\
\hline Social Ties & $\begin{array}{l}\text { Relates to friendship in on-line social networks [125], [126] and includes } \\
\text { the study of features underlying human social interactions, such as regu- } \\
\text { larity, periodicity, similarity, centrality, in the graph of social contacts of } \\
\text { individuals. }\end{array}$ & $\begin{array}{l}\text { Message propagation [77]; Influence detection; Homophily inference; Recom- } \\
\text { mendation or customised advertisement services. }\end{array}$ \\
\hline $\begin{array}{l}\text { Socio- } \\
\text { demographic } \\
\text { and socio- } \\
\text { economic traits }\end{array}$ & Relates to gender [101], age, income [111], family or marital status. & $\begin{array}{l}\text { Recommendation or customised advertisement services; incentive-based ap- } \\
\text { proaches; smartphone usage understanding; traffic profile modeling; traffic } \\
\text { engineering; urban planning. }\end{array}$ \\
\hline Traffic Profile & $\begin{array}{l}\text { Accounts for traffic volume, time periodicity, type of content, interest } \\
\text { (including application, websites, and services). }\end{array}$ & $\begin{array}{l}\text { Recommendation or customized advertisement services; traffic profile model- } \\
\text { ing; network resource allocation and optimization; load balancing. }\end{array}$ \\
\hline Others & $\begin{array}{l}\text { Any other human behavior facing their actions, habits, interests, pref- } \\
\text { erences, and context in their day-to-day life: e.g., the human inherent } \\
\text { and frequent will of sharing information (e.g., pictures, recommendations, } \\
\text { opinions, arguments, and friendship). }\end{array}$ & $\begin{array}{l}\text { On-line social networks; recommendation systems; pool transportation (e.g., } \\
\text { blablacar, uber); home-sharing (e.g., Airbnb). }\end{array}$ \\
\hline
\end{tabular}

emotions, and captures the amount of social stimuli that we search for. Agreeableness (A) describes empathy, compassion, and altruism. Neuroticism $(N)$ is associated with the tendency of experiencing negative feelings, anxiety, mood swings, and emotional instability. Personality traits levels are gathered through questionnaires built for this purpose by psychologists and available online.

Research has studied individual personality traits prediction through datasets since human-migration to the digital environment renders such prediction possible, and personality is predictive of a wide range of behavioral and social outcomes. Previous work investigates the relationship between personality and smartphone usage: i.e., if installed applications, calls, and the proximity of Bluetooth devices can lead to the prediction of the Big5 traits [131], [132].

Interactions and online social ties have also appeared to predict personality traits [120], [133]. In [120], authors reveal how Openness is correlated with check-ins at popular and social venues, while Neuroticism has a negative correlation with the number of venues visited. The connection between people's social networks, the typical locations visited, and Extraversion is studied in [134]. Finally, [135] predicts Extraversion and Conscientiousness by analyzing mobile HTTP(S) traffic during weekend nights and weekdays mornings, linking Openness to visited web pages diversity.

c) Wireless encounters: Understanding user contacts is relevant when designing new opportunistic communication protocols. In this case, the problem mainly lies in quantifying the contacts' quality according to a determined objective and correctly predicting encounters. To that end, the regularity of daily activities [113], [115], [117] applies; The tendency to follow the shortest path to a certain destination [113]; The very common short and confined traveled distances [113], [115]; The prevalence of static phases spent at a few fixed locations, with rapid transitions among those [136]; The overnight movements invariance in dwelling places with usually lower contact opportunities [116]; to cite a few.

Protocols relying on human motion estimate mobile users' potential to act as data forwarders, mainly leveraging sophisticated network analysis metrics such as centrality measures. In [35], [137], authors derive social-based metrics from users' connectivity (such as betweenness centrality and neighborhood similarity) for more efficient opportunistic forwarding decisions and less overhead. In the same way, opportunistic data offloading relying on direct communication between devices appeared in [62], [77], [88], [91]. In such works, authors (i) study how temporal communities can assist minimizing the delivery delays; (ii) investigate the node interactions through centrality measures to derive reliable future communication possibilities, or (iii) determine the copies amount to be injected in the network to ensure performance.

Finally, although an elevated rate of regularity characterizes human behavior, random events or decisions can happen. Such situations are hardly predictable and are unlikely to repeat in the future; they originate from the fact that users are reasonable beings, whose decisions they take are based on their motivations, which may also change over time [117], [130]. The goal of characterizing random and regular encounters have motivated works, such as [10]. In [10], a finer-grained classifier, is introduced to describe random and social interactions. The performed analysis unveils significant differences among the dynamics of users' interactions, which authors leveraged 
to unveil social ties' impact on opportunistic routing.

d) Social ties: Social networks map vertices to individuals, while edges may represent friendship, work interactions, similarity, among others [125]. When building such a network, edges can derive from explicit information (e.g., declared friendship on Facebook) or from implicit knowledge inferred from the reciprocate behavior of the vertices (e.g., similarity), a phenomenon called homophily in the literature of complex network analysis.

Analysis of social networks usually relies on the high predictability of human behaviors [126], [138], mostly driven by routinary activities. Hence, mechanisms such as preferential attachment and triangle formation model these networks vertices connections [139], leveraging the existence of communities or highly connected hubs in the network. This high predictability makes social networks different from random ones, such as the Erdős and Rényi network [140] (where node connections are purely stochastic, being determined by a constant probability).

Most recently, location-based social networks (LBSNs) have become relevant data sources for urban computing [101]. LBSN offers unprecedented geographic and temporal resolutions. It reflects individual user actions (temporal resolution) at the scale of entire world-class cities (global geographic resolution). For instance, users who share data in Foursquare, a popular LBSN, usually have the goal of showing to their friends where they are, while also providing personalized recommendations of places they visit. Nevertheless, when correctly analyzed for knowledge extraction, this data can suit for better understanding city dynamics and related social, economic, and cultural aspects [101], [111].

e) Content demand: Understanding individuals' content consumption is relevant when looking for solutions (i) to manage the recent boost of mobile data usage and (ii) to improve communication services quality or the design of adaptable networking protocols [141]. Such perception can help identify traffic congestion periods or fill the gap between the infrastructure technology's capacity and the mobile users' traffic load.

A significant amount of work in the literature analyzes network traffic usage through voice calls and SMS messages, both extracted from traditional Call Detail Records (CDRs) [142], [143]. Although providing valuable insights, as the CDRs present time irregularity and scarcity of call traffic, they do not describe realistic data traffic demand patterns. Browsing (visited websites) behavior has also been applied in user profiling according to their traffic demand [144]. Still, other works have categorized the actual mobile traffic usage [145], [146]. Among those, [146] provides a profiling of individual users' behavior -rather than a network-wide one and a precise temporal network usage modeling, i.e., in terms of volume as well the frequency of traffic demand - rather than only considering total volume of traffic or a normalized volume. Among the outcomes, authors show: (i) the high daywise similarity on sessions number, traffic volume, and interarrival time traffic parameters; (ii) such parameters from the same hours on different days present less variability than the parameters within the same day on different hours; (iii) the high correlation between upload/download traffic volume; (iv) peak and non-peak hours can be easily identified when it comes to users' traffic demands; (v) similar sessions number and duration occur when users are grouped by age range, irrespective of the users' gender; (vi) male participation raises as the user age increases, while the female participation decreases with the age increase.

More recently, literature works have investigated network usage concerning other users' behavior. In [146], [147], calls/SMS patterns or traffic demand of users and their sociodemographic factors (e.g., age and gender) are jointly investigated. The relation between content consumption and mobility properties is considered in studies that focus on application interests [148], data traffic dynamics [149] and service usages [150]. Finally, in [151], authors describe their investigation on the predictability of mobile data traffic generated by individual users, which is studied in isolation as well as jointly with mobility. Among the outcomes, authors show: (i) The possibility for predicting user traffic generation with an upper bound of $85 \%$; (ii) By knowing the past activities history of an individual, apart of the traffic volume, it is possible to predict where it will occur with an $88 \%$ accuracy in average. This result is possible thanks to correlations between visited locations and traffic volumes; (iii) Including location information in the prediction process allows forecasting the future consumption of mobile data traffic with $5 \%$ higher accuracy, pushing the overall performance from $85 \%$ to $90 \%$. Such results indicate a large space for predicting mobile data traffic and adapting network optimizing solutions based on the latter, such as caching and prefetching.

This section discussed the Tactful Networking paradigm, including a more granular view from aspects of humanbehavior to assist future networking solutions. Following, in the next section, we discuss a framework for enhancing raw human data with best practices to deal with challenges in this context, data source examples, and other aspects. This framework comprises data management, analytics, and privacy.

\section{Enhancing Human Raw Data to Assist COMPUTER NETWORKING SOLUTIONS}

Throughout previous sections, we discussed why humanbehavior data should become essential for future generations of mobile network architectures and models. Nevertheless, the "transformation" of raw data describing human behavior (usually collected through smart-devices or social networks) into useful knowledge requires multiple iterations.

Figure 3 depicts a general framework to process and preserve the privacy of human-behavior raw data as a means of considering the tactful networking concept in current networking architecture and paradigms. The framework features three parts, Data Management, Data Analytics, and Data Privacy, which are discussed below along with its sub-items.

\section{A. Data Management}

1) Acquisition: Human behavior analysis requires data availability, sometimes, from multiple sources (e.g., cellular networks, applications, OSNs, census, and surveys). 
Specific APIs, web crawlers, mobile crowdsensing applications, or infrastructure-based sniffers are examples of means of collecting human-related data. APIs can be streaming-based, where data is gathered almost in real-time (after some user share information) or request-based. Online social networks, like Twitter and Foursquare, make available several APIs that could be from each kind, depending on the available information, such as account activity, geo (mobility), or user posts. When there is no straight access to information (e.g., data inside HTML pages or specific databases/search engines), web crawlers are necessary. These crawlers use text mining to analyze the desired information from web pages, for example, HTML tags contents. Further, mobile devices became a common data source in the past decade. In the literature, there are two main ways of collecting mobile devices data. The first is to directly extract the sensory data from smartphones or other mobile devices [152]. The next possibility is through mobile crowdsensing datasets from special applications installed in volunteer smartphones, usually designed for research purposes. Thanks to mobile operating systems' openness, such applications can obtain diverse data from a mobile device (e.g., rotation, acceleration, GPS location, WiFi APs). On the other hand, these datasets usually have a limited number of users due to the difficulty involved in volunteer recruiting.

A secondary collection approach uses traffic logs collected from network infrastructure: i.e., WiFi or cellular networks. Operator-collected datasets, named CDRs, usually report logs of mobile network traffic demand and human footprints. CDR consists of time-stamped and geo-referenced records of voice phone calls or Internet usage of mobile network subscribers [141]. CDRs bring the benefit of encompassing huge populations (e.g., millions of users) over large (e.g., citywide or nationwide) geographic regions, and covering long periods (e.g., months or years). Nevertheless, literature has also pointed out the limitations of CDRs: They are often largely incomplete since telecommunication events are punctual and provide information at specific time instants, which are also sparse and irregularly distributed over time [153]. Some of the first datasets used for human behavior analysis came through measurements from wireless network infrastructures. Such datasets contain logs of authenticated user associations to wireless networks, most often, Campus wireless networks. Such datasets are frequently used for contact inference and opportunistic-based research [10]. Here, two individuals are assumed to be in contact if both connect to the same WiFi access point on the campus. Although providing an approximation of contacts, such a strong hypothesis has been well accepted by the research community due to the lack of large direct contact datasets available for research purposes. Other examples can be found in [154], [155].

Another potential data source is the known Big Five survey [156], introduced at Section III to infer the personality traits of users. Data traces can include the Big Five Survey answers from users and make available associated information about personality traits. The Cambridge Analytica scandal in 2018 is an example of how important the information about user personality traits can be, and how it needs protection. A personality quiz on Facebook harvested data from users and, according to investigations, was being used with malicious intentions.

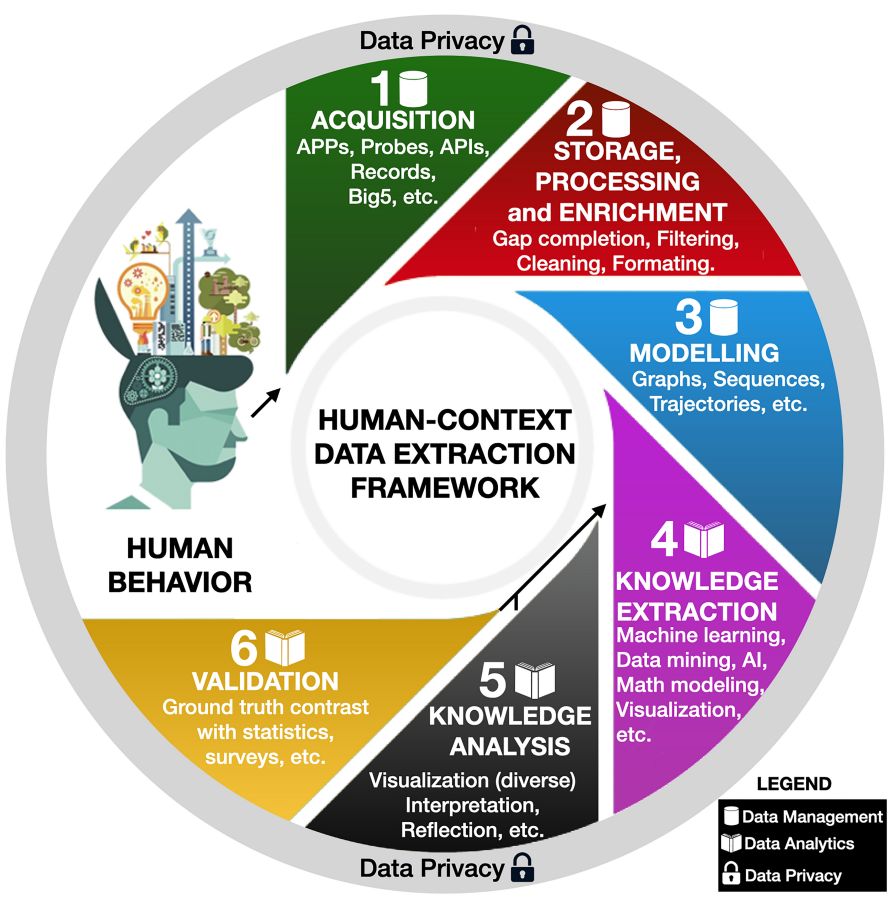

Fig. 3. Tactful Networking Framework tackling management and analysis of human-behavior information to assist future network architectures.

2) Storage, Processing, and Enrichment: After acquired, data must be stored, processed, and enriched. In human behavior information, a possibly large amount of data needs secure, scalable, and fault-tolerant storage platforms. Largescale distributed data computation is an essential aspect for data processing, as real-time and multiple parallel requests are frequent. As processing steps, associating and integrating data may be required: Where diverse data sources regrouping different data types (e.g., posts, media, location, actions, traits, and gestures) are simultaneously exploited to extract useful information. Moreover, data may present gaps, inconsistent information, semantic errors, or missing entries. Hence, data cleaning and enrichment responsible for normalization, spurious data detection, geographical and temporal interpolation, among others, may be necessary to make up missing data and to fill the gaps, while limiting biasing the raw data. As examples, we could cite processes such as time discretization, to reduce the temporal resolution of CDR Datasets and make the data appear complete [117], [129], [157], trajectory reconstruction [116], to infer positions of the users at timestamps where the original data does not provide such information, and user filtering [153], [157] to eliminate those with not enough mobility information. Finally, dimensional reduction of multidimensional data may also be necessary before analysis, where feature selection has proven effectiveness.

3) Modelling: Finally, data must be modeled in a format helping the extraction of spatio-temporal information or relation between different components of the data. Graphs have been the most used data format to model behaviors related to people's spatio-temporal association with environment and ties 
(i.e., interactions or social ties). Herein, a vertex in a graph can represent users in a network or their visited places [10], [91], [158]. At the same time, edges would connect vertex when there is an encounter between users, or when a user sequentially visits locations [159]. Spatio-temporal trajectories or time series of chronologically ordered points are other examples of formats in data modeling: e.g., modeling collected geographic data from human mobility as a set of spatiotemporal trajectories, representing data generated by a moving individual in geographical spaces [91], [112].

\section{B. Data Analytics}

Data analytics includes knowledge extraction and analysis as well as data validation, as discussed hereafter.

1) Knowledge Extraction and Analysis: These are fundamental to find new insights on data. The process should typically be continuous to foster the adaptive capability of the whole system. Understanding data properties and the kind of problem to be addressed are part of knowledge extraction. Among the types of knowledge extraction from data, we can mention pattern detection and modeling, correlation and causality among associated entities, behavior profiling, data classification or clustering, data changes or irregularity detection and modeling, to cite a few. Here, machine learning techniques, artificial intelligence, HCI methods, time series modeling, sophisticated networking metrics, statistics, and empirical analysis have become essential tools by research community performing human behavior analysis. More recently, visualization techniques [160] have become very popular: Due to the complexity of big data, such techniques make data more accessible, understandable, and usable. Among the advantages brought by visualization tools, we can mention the possibility for: quick and clear information understanding; easy identification of emerging trends allowing a fast action based on what is seen; visual identification of relationships or patterns; analysis at various levels of detail.

When it comes to human-behavior diverse spatio-temporal information, there are challenges related to data association. Discovering association and relationships between data sets, detecting unusual objects, and classifying them are not trivial tasks, particularly when considering heterogeneous data sources. Machine learning approaches are common in knowledge extraction with different intentions, such as building a scientific knowledge base with crowdsourcing to correct information [161] and filling patient data gaps into a medical decision support system [162]. Artificial Intelligence (AI) also applies to knowledge extraction and analysis. Several examples can be found, such as in [163], where authors use an ontology approach to extract meta knowledge, create metadata with the information obtained, and query metadata from RDF files to acquire the required knowledge. In [164], an AI system for big-data analysis is proposed and evaluated through a logistics company, bringing gains in productivity.

Knowledge extraction can also occur through user activity in their social networks. User posts can make available a large dataset of valuable information, such as spatial, geographic, emotional, and personality traits. Again the example from
Cambridge Analytica Scandal fits in this matter. Knowledge extraction from social networks can apply for networking resource management and user prediction [111], decentralizing networking applications and resources, among others. Data mining, natural language processing, and other techniques can be applied to extract users' information. These processes should typically be continuous with repeated iteration cycles to foster the whole system's adaptive behavior while gaining new insights, discovering mistakes, and reconsidering decisions.

2) Validation: Data validation consists of verifying data correctness and usefulness. It intends to provide guarantees for the fitness, accuracy, and consistency of any input into an application or system. A used model validation technique for assessing how the results will generalize to an independent data set is cross-validation in statistical analysis. Another widespread data validation method is the crossing of data (usually incomplete or reduced) with what is called a ground truth data (i.e., typically official or completed data). Among ground truth examples are the CENSUS data or surveys with low-error margins, or more fine-grained datasets (e.g., GPS datasets used to validate CDR trajectory reconstruction techniques). Ground truth data may not be available for all situations. In this case, repeating more experiments might be necessary, or using a simulation environment as support to validate the use of specific data in multiple scenarios or under varying conditions.

After the validation, it is necessary to shape the information for the development of services and applications. These final products are expected to help operators develop enhanced human-aware networking solutions to match user expectations more naturally.

\section{Data Privacy}

As we trod along the smart-devices, online services, and big data era, user-data privacy must be guaranteed to support applications and innovation while not harming individual rights and security. This data varies in different aspects, such as (i) quantity - guided by multiple sources and the human frequent "willingness to share" in several platforms; (ii) diversity different types and formats generated by "sensors" or acquired from indirect sources; and (iii) quality - data precision must be certified to make sure the solutions adapt correctly to their users.

In the context of Tactful Networking, user data can be available online in a distributed fashion and might get queries from multiple agents, which requires care to avoid malicious use. Previous work [165] showed that even small pieces of information linked with other data sources could reveal sensitive information that most people would like to keep private. Violations in user privacy might also lead to losses (including financial), as they bring a lack of trust in the application or system. User-data privacy and security research appears linked to different aspects, such as authentication and authorization mechanisms, anonymization, incentive, and reputation schemes. In the section II, throughout the years, several initiatives combined human aspects for reliability and privacy, such as trustworthy interactions in mobile social 
networks [95], and circles of trust from group meetings. Further, in [2], authors reviewed relevant publications linked to some of the aspects above. This section focus on recent advances in privacy-preserving big data, including data mining, management, and publishing.

User data privacy is threatened not only by attackers or by gathering information available publicly. In some instances, employees and data scientists have full access to customers' and companies' sensitive and private information. As previously stated, there is a trade-off between data utility and privacy risk. In [166], authors present a framework that allows privacy-preserving big data analytics while still providing high utility data for analysts. According to [167], due to a massive scale of diverse data generated by people, security, and privacy in Big Data still faces many challenges. They classify privacy preservation techniques in Big Data and discuss advantages and disadvantages. Finally, they present a differential privacy technique where agents (those who query the information) do not directly access the database. Within this technique, an intermediary software takes the query and adds noise to the results according to the privacy risk.

Big data publishing is also present in recent works. In [168], authors compare Datafly and Mondrian anonymization algorithms through thirteen parameters. Among those, performance, efficiency, increase in data size, and anonymization time. In [169], authors discuss the privacy problem in big data, methods to protect data publishing, evaluate big data (e.g., velocity, volume, variety, variability) from a privacy perspective, and recommendations for future research. In [170], the authors find that existing anonymization techniques fail in the trade-off between data utility and privacy. They propose a Mondrian based k-anonymity approach combined with a Deep Neural Network-based framework. Experimental results show data utility without compromising privacy.

Finally, recent work also focused on privacy-preserving data mining. In [171], authors developed a protocol to facilitate users to outsource their encrypted databases and item-set mining to a cloud environment in a privacy-preserving manner. In [172], the authors propose a privacy-preserving method for POA (place of activity) mining, using a clustering algorithm to discover POA. Differential privacy mechanisms are embedded in access to raw location data and results. The method utility is shown through location datasets derived from geo-referenced social media. In [173], an item-centric algorithm mines frequent patterns from big uncertain data. Pattern mining aims to discover implicit and potentially useful information. Existing algorithms use transaction-centric mining that is more difficult to adapt to imprecise and uncertain data. Results show algorithm effectiveness with privacy-preservation. In [174], authors discuss privacy-preserving big data management and analytics techniques in static and dynamic distributed environments, including models, issues, approaches, and reference frameworks. Complementary, in [175], they propose a framework as an alternative to classical methods that guarantee big data privacy via security-inspired protocols. The framework comprises discussions about two study-cases.

As Data Privacy is crucial for the Tactful Networking paradigm evolution, proper techniques must suit the type of data to be stored and analyzed. This section discussed recent works in Data Privacy Research. Following section features conclusions, future challenges, and research opportunities.

\section{Future Challenges and Final Considerations}

This paper discussed the Tactful Networking paradigm explaining why and how humans should increasingly participate in the communication loop of future communication standards. For such a paradigm to progress, there are multiple issues from the human, networking, and computing systems perspectives to be dealt with. First, there is a need for changing modeling practices of network solutions. In addition to the focus on network performance and metrics, it is necessary to apply big data analytics related to human context and behavior information and to bring knowledge about human behavior combining ideas and methods from different areas such as machine learning, pervasive AI, HCI, stochastic modeling, psychology, sociology, computer networks, data science, and statistics. Throughout time, correlating this data will help to analyze routines, to build enhanced incentive mechanisms, and to predict situations and behavior. This kind of information will apply to orchestrate better how the network shall behave from the operation point-of-view. The lack of publicly available rich datasets is also one of the obstacles for a better understanding of human aspects. This data also have to comprehend more significant populations, preferably full smart cities or metropolitan regions where sensing as a service will occur through billions of IoT sensors combined with cloud and edge technologies.

Second, emerging new applications, more people connected through more powerful smart-devices, cloud services massification, among others, bring data traffic raise and challenges to network core and edge. The wireless communications technologies available will deal with improved capabilities user equipment that will accelerate the proliferation of performance-hungry applications. Such challenges call for having a new architectural paradigm for the current Internet. Here, intelligence should be brought from centralized computing facilities to distributed and in-network computation. The envisaged scenario is to have network intelligence pushed at the edge, much closer to UEs, where learning, reasoning, and decision making will provide distributed autonomy, replacing the classical centralized structures: Integrating collective intelligence in the network is essential. The natural Internet upgrade into a "network of subnetworks" is thus, a new trend, where "local" evolution is the key principle to enhance situational awareness and adaptation of edge networks. Algorithms shall rely on knowledge extraction from user behavior, network heterogeneity, and uncertainty (brought by human-behavior or physical conditions of the network). As an example, by moving resources to the edge combining with human-aware knowledge can assist in fulfilling some 5G/6G requirements, innovative quality-aware services and applications, containerized micro-services, and overlay networking solutions (e.g., Kubernetes [176]).

Despite the traffic increase in the past few years, communication protocols remain limited, and, in many cases, they 
rely on strategies developed in the past, where a scenario such as the current one was not envisaged, much less the future. There is still a limited understanding of the characteristics that protocols must take into account, including the traffic carried aspects and its generation context. Thus, intelligent protocols will be required to transport the requested information at the lowest possible cost to the network, while simultaneously providing quality of service and experience for users [177]. One of the characteristics of future network architectures is that they will be used to access information and process it in a distributed way. For example, billions of $I o T$ devices that will connect through wireless at the edge of the network will need to deal with the uncertainty and unreliability of the wireless medium. Many of these devices will also have processing, battery, and memory limitations. Therefore, the software platforms and the network protocol stack executed in them must take the user into consideration.

Even with a vast literature available, there are still gaps in predicting human behavior under the influence of psychological, social, and demographic factors, among others, that should impact prediction models [178]. Quantitative studies are needed to uncover an expected degree or precision of these learning and predictions, which more suitable techniques to predict individual's behavior, and how factors such as those above interfere with accuracy. Previous prediction methods require an extensive data history and high regularity of events. This fact reinforces the need to make datasets available and makes urgent the design of prediction techniques providing high accuracy while based on limited datasets.

Understanding better the human decision-making process is also necessary, as the decisions we take can reflect in extracted human-traits increasingly used as input to develop networking solutions. According to [127], "a group of individuals, no matter how highly organized it may be at any given instant, tends toward greater disorder or randomization, called entropy". Therefore, investigating and detecting what causes entropy, when, and why it happens on human decisionmaking is essential for future Tactful Networking solutions. The amount of individual entropy can also link to aspects such as Personality Traits, which influence other human traits like mobility patterns, interactions, and social activities. Nevertheless, according to [127], Intrinsic Information Theory (IIT) is useful to understand the conscious state of mind on decisionmaking. Consciousness is led by aspects such as spatial and temporal boundaries, information, and individual perspectives. Establishing this relationship with decision-making is essential, as we call for future network design to take human behavioral aspects into account. Our behavior and individual characteristics impact directly on how we demand network resources as well as what kind of resources are requested.

In today's scenarios with a diversity of devices, users' behaviors and requirements, conventional Internet solutions are no more adapted. Recent works in the literature considered different aspects of human behavior and context to achieve better performance [91], [179]. One of the challenges will be identifying what kind of context information and human behavior can be extracted and leveraged by Internet solutions to favor resources management and user quality of service and experience.

The Tactful Networking paradigm will also be a cornerstone for envisaged $6 \mathrm{G}$ that will empower our cities to be smart and fully connected with a multitude of services and devices. 6G will also be boosted by Deep Learning from Artificial Intelligence (AI) relying on data mining from network aspects and user devices and behavior to provide contextaware service-enabled communications. A human-aware AI layer will enable more intelligent network services deployment and empower applications such as autonomous driving and connected vehicles, haptic communications, augmented reality, smart healthcare, and smart homes. Concerning 6G challenges, the study of human aspects can bring insights to future edge and cloud computing, higher networking densification (with even smaller cells to reduce latency and increase capacity), and dynamic topology environments.

Finally, whenever human user data is being collected, stored, or analyzed, suitable privacy-preserving mechanisms must be provided. Future Tactful Networking solutions must find the balance between privacy and user satisfaction, and among privacy and protocols, services, and application utility. These solutions or services must keep in mind that there will be no privacy implementation in real life if users are cut from their typical applications, services, or likes. Yet, although GDPR (General Data Protection Regulation) [180] gives users legal privacy grounds, centralized data management increase the appeal for attacks on their facilities (e.g., a single breach led to millions of users data stored in big cloud infrastructures) [181]. Instead, distributed management inherently disperses valuable information and facilitates the use of privateowned data management systems [180]. Therefore, distributed edge servers are much less likely to become the target of security attacks, reducing information leakage.

\section{REFERENCES}

[1] I. F. Akyildiz, S. Nie, S.-C. Lin, and M. Chandrasekaran, "5g roadmap: 10 key enabling technologies," Comput. Netw., vol. 106, no. Supplement C, pp. $17-48,2016$.

[2] L. Duan, L. Huang, C. Langbort, A. Pozdnukhov, J. Walrand, and L. Zhang, "Human-in-the-loop mobile networks: A survey of recent advancements," IEEE Journal on Selected Areas in Commun., vol. 35 , no. 4, pp. 813-831, Apr. 2017.

[3] K. Jaffrès-Runser, G. Jakllari, T. Peng, and V. Nitu, "Crowdsensing mobile content and context data: Lessons learned in the wild," in Proc. IEEE PERCOM Workshops, Mar. 2017, pp. 311-315.

[4] Q. Yan, W. Chen, and H. V. Poor, "Big data driven wireless communications: A human-in-the-loop pushing technique for $5 \mathrm{~g}$ systems," IEEE Wireless Commun., vol. 25, no. 1, pp. 64-69, Feb. 2018.

[5] R. Sofia and P. Mendes, "User-provided networks: consumer as provider," IEEE ComMag, vol. 46, no. 12, pp. 86-91, Dec. 2008.

[6] R. Bolla, R. Rapuzzi, and M. Repetto, "Handling mobility over the network," in Proc. ACM CFI, 2009, pp. 16-19.

[7] S. Vihavainen, A. Oulasvirta, and R. Sarvas, "I can't lie anymore!: The implications of location automation for mobile social applications," in Proc. IEEE MobiQuitous, Jul. 2009, pp. 1-10.

[8] G. Biczók, L. Toka, A. Vidacs, and T. A. Trinh, "On incentives in global wireless communities," in Proc. ACM U-NET Workshop, 2009, pp. 1-6.

[9] A. Mislove, B. Viswanath, K. P. Gummadi, and P. Druschel, "You are who you know: Inferring user profiles in online social networks," in Proc. ACM WSDM, 2010, pp. 251-260.

[10] P. Olmo Vaz de Melo, A. Carneiro Viana, M. Fiore, K. Jaffrès-Runser, F. Le Mouël, A. A. F. Loureiro, L. Addepalli, and G. Chen, "RECAST: Telling Apart Social and Random Relationships in Dynamic Networks," Performance Evaluation, vol. 87, no. 19-36, pp. 1-1, Mar. 2015. 
[11] T. Hossmann, T. Spyropoulos, and F. Legendre, "Know thy neighbor: Towards optimal mapping of contacts to social graphs for dtn routing," in Proc. IEEE INFOCOM, Mar. 2010, pp. 1-9.

[12] M. Balman, E. Chaniotakisy, A. Shoshani, and A. Sim, "A flexible reservation algorithm for advance network provisioning," in Proc. ACM/IEEE SC, Nov. 2010, pp. 1-11.

[13] A. Sehgal and R. Agrawal, "Qos based network selection scheme for 4g systems," IEEE Trans. on Consumer Elect., vol. 56, no. 2, pp. 560565, May 2010.

[14] J. Antoniou, V. Papadopoulou, V. Vassiliou, and A. Pitsillides, "Cooperative user-network interactions in next generation communication networks," Comput. Netw., vol. 54, no. 13, pp. 2239 - 2255, 2010.

[15] A. G. Ribeiro, R. Sofia, and A. Zúquete, "Improving mobile networks based on social mobility modeling," in Proc IEEE ICNP, Oct 2011, pp. 289-291.

[16] J. Cho, A. Swami, and I. Chen, "A survey on trust management for mobile ad hoc networks," IEEE Commun. Surveys Tutorials, vol. 13, no. 4, pp. 562-583, 2011

[17] D. Christin, A. Reinhardt, S. S. Kanhere, and M. Hollick, "A survey on privacy in mobile participatory sensing applications," Journal of Systems and Software, vol. 84, no. 11, pp. 1928 - 1946, 2011.

[18] E. Ibarrola, J. Xiao, F. Liberal, and A. Ferro, "Internet qos regulation in future networks: a user-centric approach," IEEE ComMag, vol. 49, no. 10, pp. 148-155, Oct 2011.

[19] J. Márquez-Barja, C. T. Calafate, J.-C. Cano, and P. Manzoni, "An overview of vertical handover techniques: Algorithms, protocols and tools," Comput. Commun., vol. 34, no. 8, pp. 985 - 997, 2011.

[20] A. Jimenez-Molina and I.-Y. Ko, "Spontaneous task composition in urban computing environments based on social, spatial, and temporal aspects," Engineering Appl. of Artif. Intelligence, vol. 24, no. 8, pp. 1446 - 1460, 2011.

[21] Y. Lu, M. Motani, and W.-C. Wong, "The user-context module: A new perspective on future internet design," Procedia Comput. Science, vol. 5, pp. $280-287,2011$.

[22] A. Tudzarov and T. Janevski, "Functional architecture for $5 \mathrm{~g}$ mobile networks," Int. Journal of Adv. Science and Technol., vol. 3, 082011.

[23] R. Sofia, P. Mendes, M. Damásio, S. Henriques, F. Giglietto, E. Giambitto, and A. Bogliolo, "Moving towards a socially-driven internet architectural design," Proc. ACM SIGCOMM, vol. 42, 072012.

[24] E. Palomar, A. Alcaide, A. Ribagorda, and Y. Zhang, "The peer's dilemma: A general framework to examine cooperation in pure peerto-peer systems," Comput. Netw., vol. 56, no. 17, pp. 3756 - 3766, 2012.

[25] M. Yildiz, M. A. Khan, F. Sivrikaya, and S. Albayrak, "Cooperation incentives based load balancing in ucn: A probabilistic approach," in Proc. IEEE GLOBECOM, Dec 2012, pp. 2746-2752.

[26] S. Zarifzadeh, N. Yazdani, and A. Nayyeri, "Energy-efficient topology control in wireless ad hoc networks with selfish nodes," Comput. Netw., vol. 56, no. 2, pp. $902-914,2012$.

[27] C. Huang, G. M. Lee, and N. Crespi, "A semantic enhanced service exposure model for a converged service environment," IEEE ComMag, vol. 50, no. 3, pp. 32-40, Mar. 2012.

[28] H. Tuncer, S. Mishra, and N. Shenoy, "A survey of identity and handoff management approaches for the future internet," Comput. Commun., vol. 36, no. 1, pp. 63 - 79, 2012.

[29] Y. Najaflou, B. Jedari, F. Xia, L. T. Yang, and M. S. Obaidat, "Safety challenges and solutions in mobile social networks," IEEE Systems Journal, vol. 9, no. 3, pp. 834-854, Sep. 2013.

[30] M. Yildiz and M. A. Khan, "Mobility behavior modeling in ucn," in Proc. IEEE WCNC, Apr. 2013, pp. 2184-2189.

[31] P. Bellavista, R. Montanari, and S. K. Das, "Mobile social networking middleware: A survey," Pervasive and Mobile Comput., vol. 9, no. 4, pp. $437-453,2013$.

[32] M. Amani, A. Aijaz, N. Uddin, and A. H. Aghvami, "On mobile data offloading policies in heterogeneous wireless networks," in Proc. IEEE VTC Spring, Jun. 2013, pp. 1-5.

[33] M. Conti, M. Mordacchini, and A. Passarella, "Design and performance evaluation of data dissemination systems for opportunistic networks based on cognitive heuristics," ACM Trans. Auton. Adapt. Syst., vol. 8, no. 3, pp. 12:1-12:32, Sep. 2013.

[34] J. Wu, M. Xiao, and L. Huang, "Homing spread: Community homebased multi-copy routing in mobile social networks," in Proc. IEEE INFOCOM, Apr. 2013, pp. 2319-2327.

[35] Y. Zhu, B. Xu, X. Shi, and Y. Wang, "A survey of social-based routing in delay tolerant networks: Positive and negative social effects," IEEE Commun. Surveys Tutorials, vol. 15, no. 1, pp. 387-401, First 2013.
[36] Y. Chen and Y. Wei, "A beacon-based trust management system for enhancing user centric location privacy in vanets," Journal of Commun. and Netw., vol. 15, no. 2, pp. 153-163, Apr. 2013.

[37] T. H. Silva, P. O. S. V. de Melo, A. C. Viana, J. M. Almeida, J. Salles, and A. A. F. Loureiro, "Traffic condition is more than colored lines on a map: Characterization of waze alerts," in Social Informatics. Springer, 2013, pp. 309-318.

[38] X. Li, O. Mehani, R. Agüero, U. Toseef, Y. Zaki, and C. Görg, "Evaluating user-centric multihomed flow management in multi-user scenarios," in Proc. IEEE WoWMoM, Jun. 2013, pp. 1-7.

[39] G. Aloi, M. D. Felice, V. Loscrì, P. Pace, and G. Ruggeri, "Spontaneous smartphone networks as a user-centric solution for the future internet," IEEE ComMag, vol. 52, no. 12, pp. 26-33, Dec. 2014.

[40] T. Jamal and P. Mendes, "Cooperative relaying in user-centric networking under interference conditions," IEEE ComMag, vol. 52, no. 12, pp. 18-24, Dec. 2014.

[41] M. N. Tehrani, M. Uysal, and H. Yanikomeroglu, "Device-to-device communication in $5 \mathrm{~g}$ cellular networks: challenges, solutions, and future directions," IEEE ComMag, vol. 52, no. 5, pp. 86-92, May 2014

[42] B. Bangerter, S. Talwar, R. Arefi, and K. Stewart, "Networks and devices for the 5g era," IEEE ComMag, vol. 52, no. 2, pp. 90-96, Feb. 2014.

[43] C. I, C. Rowell, S. Han, Z. Xu, G. Li, and Z. Pan, "Toward green and soft: a $5 \mathrm{~g}$ perspective," IEEE ComMag, vol. 52, no. 2, pp. 66-73, Feb. 2014.

[44] X. Xing, T. Jing, W. Zhou, X. Cheng, Y. Huo, and H. Liu, "Routing in user-centric networks," IEEE ComMag, vol. 52, no. 9, pp. 44-51, Sep. 2014.

[45] A. Ferragut and F. Paganini, "Network resource allocation for users with multiple connections: Fairness and stability," IEEE/ACM Trans. on Netw., vol. 22, no. 2, pp. 349-362, Apr. 2014.

[46] P. A. Frangoudis and G. C. Polyzos, "Security and performance challenges for user-centric wireless networking," IEEE ComMag, vol. 52, no. 12 , pp. $48-55$, Dec. 2014

[47] T. Winkler and B. Rinner, "Security and privacy protection in visual sensor networks: A survey," ACM Comput. Surv., vol. 47, no. 1, pp. 2:1-2:42, May 2014.

[48] F. Mezghani, R. Dhaou, M. Nogueira, and A. Beylot, "Content dissemination in vehicular social networks: taxonomy and user satisfaction," IEEE ComMag, vol. 52, no. 12, pp. 34-40, Dec. 2014.

[49] G. Iosifidis, L. Gao, J. Huang, and L. Tassiulas, "Incentive mechanisms for user-provided networks," IEEE ComMag, vol. 52, no. 9, pp. 20-27, Sep. 2014

[50] G. Lee, H. Kim, Y. Cho, and S. Lee, "Qoe-aware scheduling for sigmoid optimization in wireless networks," IEEE Commun. Letters, vol. 18, no. 11, pp. 1995-1998, Nov 2014.

[51] M. Katsarakis, G. Fortetsanakis, P. Charonyktakis, A. Kostopoulos, and M. Papadopouli, "On user-centric tools for qoe-based recommendation and real-time analysis of large-scale markets," IEEE ComMag, vol. 52, no. 9 , pp. 37-43, Sep. 2014.

[52] B. A. A. Nunes, M. A. S. Santos, B. T. de Oliveira, C. B. Margi, K. Obraczka, and T. Turletti, "Software-defined-networking-enabled capacity sharing in user-centric networks," IEEE ComMag, vol. 52, no. 9 , pp. $28-36$, Sep. 2014

[53] V. F. Mota, F. D. Cunha, D. F. Macedo, J. M. Nogueira, and A. A. Loureiro, "Protocols, mobility models and tools in opportunistic networks: A survey," Comput. Commun., vol. 48, pp. 5 - 19, 2014, opportunistic networks.

[54] M. Conti and S. Giordano, "Mobile ad hoc networking: milestones, challenges, and new research directions," IEEE ComMag, vol. 52, no. 1, pp. 85-96, Jan. 2014.

[55] M. Eslami, O. Karimi, and T. Khodadadi, "A survey on wireless mesh networks: Architecture, specifications and challenges," in Proc. IEEE ICSGRC, Aug 2014, pp. 219-222.

[56] R. Schoenen and H. Yanikomeroglu, "User-in-the-loop: spatial and temporal demand shaping for sustainable wireless networks," IEEE ComMag, vol. 52, no. 2, pp. 196-203, Feb. 2014.

[57] D. S. Nunes, P. Zhang, and J. Sá Silva, "A survey on human-in-theloop applications towards an internet of all," IEEE Commun. Surveys Tutorials, vol. 17, no. 2, pp. 944-965, Secondquarter 2015.

[58] Z. Wang, R. Schoenen, H. Yanikomeroglu, and M. St-Hilaire, "Load balancing in cellular networks with user-in-the-loop: A spatial traffic shaping approach," in Proc. IEEE ICC, Jun. 2015, pp. 2638-2643.

[59] D. Syrivelis, G. Iosifidis, D. Delimpasis, K. Chounos, T. Korakis, and L. Tassiulas, "Bits and coins: Supporting collaborative consumption of mobile internet," in Proc. IEEE INFOCOM, Apr. 2015, pp. 2146-2154. 
[60] P. Castagno, R. Gaeta, M. Grangetto, and M. Sereno, "Device-to-device content distribution in cellular networks: A user-centric collaborative strategy," in Proc. IEEE GLOBECOM, Dec 2015, pp. 1-6.

[61] P. Ameigeiras, J. J. Ramos-munoz, L. Schumacher, J. Prados-Garzon, J. Navarro-Ortiz, and J. M. Lopez-soler, "Link-level access cloud architecture design based on sdn for 5g networks," IEEE Network, vol. 29, no. 2, pp. 24-31, Mar. 2015.

[62] F. Rebecchi, M. Dias de Amorim, V. Conan, A. Passarella, R. Bruno, and M. Conti, "Data offloading techniques in cellular networks: A survey," IEEE Commun. Surveys Tutorials, vol. 17, no. 2, pp. 580603, 2015.

[63] D. Montero, M. Yannuzzi, A. Shaw, L. Jacquin, A. Pastor, R. SerralGracia, A. Lioy, F. Risso, C. Basile, R. Sassu, M. Nemirovsky, F. Ciaccia, M. Georgiades, S. Charalambides, J. Kuusijarvi, and F. Bosco, "Virtualized security at the network edge: a user-centric approach," IEEE ComMag, vol. 53, no. 4, pp. 176-186, Apr. 2015.

[64] H. Mousa, S. B. Mokhtar, O. Hasan, O. Younes, M. Hadhoud, and L. Brunie, "Trust management and reputation systems in mobile participatory sensing applications: A survey," Comput. Netw., vol. 90, pp. $49-73,2015$.

[65] X. Hu, T. H. S. Chu, V. C. M. Leung, E. C. . Ngai, P. Kruchten, and H. C. B. Chan, "A survey on mobile social networks: Applications, platforms, system architectures, and future research directions," IEEE Commun. Surveys Tutorials, vol. 17, no. 3, pp. 1557-1581, 2015.

[66] Y. Wang and X. Lin, "User-provided networking for qoe provisioning in mobile networks," IEEE Wireless Commun., vol. 22, no. 4, pp. 26 33, Aug. 2015.

[67] F. Delmastro, V. Arnaboldi, and M. Conti, "People-centric computing and communications in smart cities," IEEE ComMag, vol. 54, no. 7 , pp. 122-128, Jul. 2016.

[68] C. I, S. Han, Z. Xu, S. Wang, Q. Sun, and Y. Chen, "New paradigm of $5 \mathrm{~g}$ wireless internet," IEEE Journal on Selected Areas in Commun., vol. 34, no. 3, pp. 474-482, Mar. 2016.

[69] M. Kamel, W. Hamouda, and A. Youssef, "Ultra-dense networks: A survey," IEEE Commun. Surveys Tutorials, vol. 18, no. 4, pp. 2522 2545, 2016.

[70] J. Park, S. Y. Jung, S. Kim, M. Bennis, and M. Debbah, "User-centric mobility management in ultra-dense cellular networks under spatiotemporal dynamics," in Proc. IEEE GLOBECOM, Dec 2016, pp. 1-6.

[71] R. Shokri, G. Theodorakopoulos, and C. Troncoso, "Privacy games along location traces: A game-theoretic framework for optimizing location privacy," ACM Trans. Priv. Secur., vol. 19, no. 4, pp. 11:111:31, Dec. 2016.

[72] V. Arnaboldi, M. Conti, M. La Gala, A. Passarella, and F. Pezzoni, "Ego network structure in online social networks and its impact on information diffusion," Comput. Commun., vol. 76, no. C, pp. 26-41, Feb. 2016.

[73] P. Charonyktakis, M. Plakia, I. Tsamardinos, and M. Papadopouli, "On user-centric modular qoe prediction for voip based on machine-learning algorithms," IEEE Trans. on Mobile Comput., vol. 15, no. 6, pp. $1443-$ 1456, Jun. 2016.

[74] S. CC, V. Raychoudhury, G. Marfia, and A. Singla, "A survey of routing and data dissemination in delay tolerant networks," J. Netw. Comput. Appl., vol. 67, no. C, pp. 128-146, May 2016.

[75] J. Ding, L. Jiang, and C. He, "Energy-efficient power control for underlaying d2d communication with channel uncertainty: User-centric versus network-centric," Journal of Commun. and Netw., vol. 18, no. 4, pp. 589-599, Aug 2016.

[76] K. I. Pedersen, M. Niparko, J. Steiner, J. Oszmianski, L. Mudolo, and S. R. Khosravirad, "System level analysis of dynamic user-centric scheduling for a flexible 5g design," in Proc. IEEE GLOBECOM, Dec 2016, pp. 1-6.

[77] I. O. Nunes, P. O. S. Vaz de Melo, and A. A. F. Loureiro, "Leveraging d2d multihop communication through social group meeting awareness," IEEE Wireless Commun., vol. 23, no. 4, pp. 12-19, Aug. 2016.

[78] C. O. Rolim, A. G. Rossetto, V. R. Leithardt, G. A. Borges, C. F Geyer, T. F. dos Santos, and A. M. Souza, "Situation awareness and computational intelligence in opportunistic networks to support the data transmission of urban sensing applications," Comput. Netw., vol. 111, no. C, pp. 55-70, Dec. 2016.

[79] Q. Yan, W. Chen, B. Bai, and H. V. Poor, "Multicast-pushing with human-in-the-loop: Where social networks meet wireless communications," in Proc. IEEE ICC, May 2017, pp. 1-6.

[80] P. Gandotra, R. K. Jha, and S. Jain, "A survey on device-to-device (d2d) communication: Architecture and security issues," Journal of Netw. and Comput. Appl., vol. 78, no. Supplement C, pp. 9 - 29, 2017.
[81] M. Kamel, W. Hamouda, and A. Youssef, "Performance analysis of multiple association in ultra-dense networks," IEEE Trans. on Commun., vol. 65, no. 9, pp. 3818-3831, Sep. 2017.

[82] S. Wang, X. Zhang, Y. Zhang, L. Wang, J. Yang, and W. Wang, "A survey on mobile edge networks: Convergence of computing, caching and communications," IEEE Access, vol. 5, pp. 6757-6779, 2017.

[83] S. Barmpounakis, A. Kaloxylos, P. Spapis, and N. Alonistioti, "Context-aware, user-driven, network-controlled rat selection for $5 \mathrm{~g}$ networks," Comput. Netw., vol. 113, no. C, pp. 124-147, Feb. 2017.

[84] M. Shafi, A. F. Molisch, P. J. Smith, T. Haustein, P. Zhu, P. De Silva, F. Tufvesson, A. Benjebbour, and G. Wunder, "5g: A tutorial overview of standards, trials, challenges, deployment, and practice," IEEE Journal on Selected Areas in Commun., vol. 35, no. 6, pp. 12011221, Jun. 2017.

[85] G. Iosifidis, L. Gao, J. Huang, and L. Tassiulas, "Efficient and fair collaborative mobile internet access," IEEE/ACM Trans. on Netw., vol. 25 , no. 3, pp. 1386-1400, Jun. 2017.

[86] J. Ma, J. Wang, and P. Fan, "A cooperation-based caching scheme for heterogeneous networks," IEEE Access, vol. 5, pp. 15 013-15020, 2017.

[87] N. Cruz and H. Miranda, "Recurring contacts between groups of devices: Analysis and application," IEEE Trans. on Mobile Comput., vol. 17, no. 7, pp. 1666-1679, Jul. 2018.

[88] Y. Meng, C. Jiang, H. Chen, and Y. Ren, "Cooperative device-todevice communications: Social networking perspectives," IEEE Network, vol. 31, no. 3, pp. 38-44, May 2017.

[89] D. Koll, J. Li, and X. Fu, "The good left undone: Advances and challenges in decentralizing online social networks," Comput. Commun., vol. 108 , pp. $36-51,2017$.

[90] D. Tsolkas, E. Liotou, N. Passas, and L. Merakos, "A survey on parametric qoe estimation for popular services," Journal of Netw. and Comput. Appl., vol. 77, 012017.

[91] I. O. Nunes, C. Celes, I. Nunes, P. O. S. Vaz de Melo, and A. A. F. Loureiro, "Combining spatial and social awareness in d2d opportunistic routing," IEEE ComMag, vol. 56, no. 1, pp. 128-135, Jan 2018.

[92] T. Qiu, N. Chen, K. Li, M. Atiquzzaman, and W. Zhao, "How can heterogeneous internet of things build our future: A survey," IEEE Commun. Surveys Tutorials, vol. 20, no. 3, pp. 2011-2027, 2018.

[93] Q. Yan, W. Chen, and H. V. Poor, "Big data driven wireless communications: A human-in-the-loop pushing technique for $5 \mathrm{~g}$ systems," IEEE Wireless Commun., vol. 25, no. 1, pp. 64-69, Feb. 2018.

[94] D. Wang, Y. Lan, T. Zhao, Z. Yin, and X. Wang, "On the design of computation offloading in cache-aided d2d multicast networks," IEEE Access, vol. 6, pp. 63 426-63 441, 2018.

[95] E. K. Wang, Y. Li, Y. Ye, S. M. Yiu, and L. C. K. Hui, "A dynamic trust framework for opportunistic mobile social networks," IEEE Trans. on Network and Service Management, vol. 15, no. 1, pp. 319-329, Mar. 2018.

[96] C. Xu, M. Wang, X. Chen, L. Zhong, and L. A. Grieco, "Optimal information centric caching in $5 \mathrm{~g}$ device-to-device communications," IEEE Trans. on Mobile Comput., vol. 17, no. 9, pp. 2114-2126, Sep. 2018.

[97] J. Yan, D. Wu, H. Wang, and R. Wang, "User centric content sharing based on $\mathrm{d} 2 \mathrm{~d}$ cellular networks," IEEE Trans. on Vehicular Technol., vol. 67, no. 11, pp. 11208-11218, Nov 2018.

[98] A. Aijaz, "Toward human-in-the-loop mobile networks: A radio resource allocation perspective on haptic communications," IEEE Trans. on Wireless Commun., vol. 17, no. 7, pp. 4493-4508, Jul. 2018.

[99] G. Zhang, F. Ke, Y. Peng, C. Zhang, and H. Zhang, "User access and resource allocation in full-duplex user-centric ultra-dense heterogeneous networks," in Proc. IEEE GLOBECOM, Dec 2018, pp. 1-6.

[100] S. Zhang, P. He, K. Suto, P. Yang, L. Zhao, and X. Shen, "Cooperative edge caching in user-centric clustered mobile networks," IEEE Trans. on Mobile Comput., vol. 17, no. 8, pp. 1791-1805, Aug 2018.

[101] T. H. Silva, A. C. Viana, F. Benevenuto, L. Villas, J. Salles, A. Loureiro, and D. Quercia, "Urban computing leveraging location-based social network data: A survey," ACM Comput. Surv., vol. 52, no. 1, pp. 17:117:39, Feb. 2019.

[102] Y. Yang, X. Deng, D. He, Y. You, and R. Song, "Machine learning inspired codeword selection for dual connectivity in $5 \mathrm{~g}$ user-centric ultra-dense networks," IEEE Trans. on Vehicular Technol., vol. 68 , no. 8, pp. 8284-8288, Aug 2019.

[103] A. S. Shafigh, S. Glisic, E. Hossain, B. Lorenzo, and L. A. DaSilva, "User-centric distributed spectrum sharing in dynamic network architectures," IEEE/ACM Trans. on Netw., vol. 27, no. 1, pp. 15-28, Feb 2019. 
[104] S. Zaidi, O. Ben Smida, S. Affes, U. Vilaipornsawai, L. Zhang, and P. Zhu, "User-centric base-station wireless access virtualization for future 5g networks," IEEE Trans. on Commun., vol. 67, no. 7, pp. 5190-5202, Jul. 2019.

[105] H. Zhang, W. Huang, and Y. Liu, "Handover probability analysis of anchor-based multi-connectivity in $5 \mathrm{~g}$ user-centric network," IEEE Wireless Commun. Letters, vol. 8, no. 2, pp. 396-399, Apr. 2019.

[106] W. Saad, M. Bennis, and M. Chen, "A vision of $6 \mathrm{~g}$ wireless systems: Applications, trends, technologies, and open research problems," IEEE Network, vol. 34, no. 3, pp. 134-142, 2020.

[107] K. B. Letaief, W. Chen, Y. Shi, J. Zhang, and Y. A. Zhang, "The roadmap to 6g: Ai empowered wireless networks," IEEE Communications Magazine, vol. 57, no. 8, pp. 84-90, 2019.

[108] Cisco, "Annual internet report (2018-2023)," [Online]. Available: $\quad$ https://www.cisco.com/c/en/us/solutions/executiveperspectives/annual-internet-report/, 2020.

[109] K. Antonakoglou, X. Xu, E. Steinbach, T. Mahmoodi, and M. Dohler "Toward haptic communications over the $5 \mathrm{~g}$ tactile internet," IEEE Commun. Surveys \& Tutorials, vol. 20, no. 4, pp. 3034-3059, 2018.

[110] A. Aijaz and M. Sooriyabandara, "The tactile internet for industries: A review," Proc. of the IEEE, vol. 107, no. 2, pp. 414-435, 2019.

[111] K. Thilakarathna, S. Seneviratne, K. Gupta, M. A. Kaafar, and A. Seneviratne, "A deep dive into location-based communities in social discovery networks," Comput. Commun., vol. 100, 112016.

[112] C. Comito, D. Falcone, and D. Talia, "Mining human mobility patterns from social geo-tagged data," Pervasive and Mobile Comput., vol. 33 , pp. $91-107,2016$.

[113] E. M. R. Oliveira, A. C. Viana, C. Sarraute, J. Brea, and I. AlvarezHamelin, "On the regularity of human mobility," Pervasive and Mobile Computing, vol. 33, no. Supplement C, pp. 73 - 90, 2016.

[114] G. Zyba, G. M. Voelker, S. Ioannidis, and C. Diot, "Dissemination in opportunistic mobile ad-hoc networks: The power of the crowd," in Proc. IEEE INFOCOM, Apr. 2011, pp. 1179-1187.

[115] M. C. González, C. A. Hidalgo, and A.-L. Barabási, "Understanding individual human mobility patterns," Nature, vol. 453, no. 7196, pp. 779-782, Jun. 2008.

[116] G. Chen, S. Hoteit, A. C. Carneiro Viana, M. Fiore, and C. Sarraute, "Enriching sparse mobility information in Call Detail Records," Comput. Commun., vol. 122, pp. 44-58, 2018.

[117] C. Song, Z. Qu, N. Blumm, and A.-L. Barabasi, "Limits of predictability in human mobility," Science, vol. 327, no. 5968, pp. 1018-1021, Feb. 2010.

[118] I. F. Akyildiz, P. Wang, and S.-C. Lin, "Softair: A software defined networking architecture for $5 \mathrm{~g}$ wireless systems," Comput. Netw. vol. 85, pp. $1-18,2015$

[119] B. Raad and B. Mlacic, Big Five Factor Model, Theory and Structure. US: Elsevier, 12 2015, pp. 559-566.

[120] M. J. Chorley, R. M. Whitaker, and S. M. Allen, "Personality and location-based social networks," Comput. in Human Behavior, vol. 46 pp. 45-56, 2015

[121] J. Staiano, F. Pianesi, B. Lepri, N. Sebe, N. Aharony, and A. Pentland, "Friends don't lie," in Proc. ACM UbiComp, 2012.

[122] A. Paudel, B. R. Bajracharya, M. Ghimire, N. Bhattarai, and D. S. Baral, "Using personality traits information from social media for music recommendation," in Proc. IEEE ICCCS, Oct 2018, pp. 116121.

[123] H. Ning, S. Dhelim, and N. Aung, "Personet: Friend recommendation system based on big-five personality traits and hybrid filtering," IEEE Trans. on Comput. Social Systems, vol. 6, no. 3, pp. 394-402, Jun. 2019

[124] R. D. Oliveira, M. Cherubini, and N. Oliver, "Influence of personality on satisfaction with mobile phone services," ACM Trans. Comput.Hum. Interact., vol. 20, no. 2, pp. 10:1-10:23, May 2013.

[125] L. Backstrom, D. Huttenlocher, J. Kleinberg, and X. Lan, "Group formation in large social networks: membership, growth, and evolution," in Proc. ACM SIGKDD, 2006, pp. 44-54.

[126] M. Buchanan, "Behavioural Science: Secret signals," Nature, vol. 457, no. 7229, pp. 528-530, Jan. 2009.

[127] J. Rodger, "Quantumis: A qualia consciousness awareness and information theory quale approach to reducing strategic decision-making entropy," Entropy, vol. 21, p. 125, 012019.

[128] C. M. Schneider, V. Belik, and M. C. G. Thomas Couronné, Zbigniew Smoreda, "Unravelling daily human mobility motifs journal of the royal society interface," Journal of The Royal Society Interface, vol. 10, no. 84, p. 1, 2013.
[129] Y.-A. Montjoye, C. Hidalgo, M. Verleysen, and V. Blondel, "Unique in the crowd: The privacy bounds of human mobility," Scientific reports, vol. 3, p. 1376, 032013.

[130] A. Cuttone, S. Lehmann, and M. C. González, "Understanding predictability and exploration in human mobility," EPJ Data Science, vol. 7, no. 1, p. 2, Jan 2018.

[131] Y.-A. de Montjoye, J. Quoidbach, F. Robic, and A. S. Pentland, "Predicting personality using novel mobile phone-based metrics," in Social Comput., Behavioral-Cultural Modeling and Prediction, ser. 1, no. 1,2013 , pp. $48-55$.

[132] B. Mønsted, A. Mollgaard, and J. Mathiesen, "Phone-based metric as a predictor for basic personality traits," Journal of Research in Personality, vol. 74, pp. 16-22, 2018.

[133] F. Alam, E. A. Stepanov, and G. Riccardi, "Personality traits recognition on social network-Facebook," in Proc. ICWSM, 2013.

[134] L. Alessandretti, P. Sapiezynski, V. Sekara, S. Lehmann, and A. Baronchelli, "Evidence for a conserved quantity in human mobility," Nature Human Behaviour, vol. 1, no. 1, pp. 1-1, 2018.

[135] S. Park, A. Matic, K. Garg, and N. Oliver, "When simpler data does not imply less information: A study of user profiling scenarios with constrained view of mobile HTTP(S) traffic," ACM Trans. Web, vol. 12, no. 2, pp. 9:1-9:23, 2018

[136] M. Ficek and L. Kencl, "Inter-call mobility model: A spatio-temporal refinement of call data records using a gaussian mixture model," in Proc. IEEE INFOCOM, 2012, pp. 469-477.

[137] P. Hui, J. Crowcroft, and E. Yoneki, "Bubble rap: Social-based forwarding in delay-tolerant networks," IEEE Trans. on Mobile Comput., vol. 10, no. 11, pp. 1576-1589, Nov 2011.

[138] E. Cho, S. A. Myers, and J. Leskovec, "Friendship and mobility: user movement in location-based social networks," in Proc. ACM SIGKDD, 2011, pp. 1082-1090.

[139] J. Leskovec, L. Backstrom, R. Kumar, and A. Tomkins, "Microscopic evolution of social networks," in Proc. ACM SIGKDD, 2008, pp. 462 470.

[140] P. Erdös and A. Rényi, "On the evolution of random graphs," Publ. Math. Inst. Hung. Acad. Sci., vol. 7, p. 17, 1960

[141] D. Naboulsi, M. Fiore, S. Ribot, and R. Stanica, "Large-scale Mobile Traffic Analysis: a Survey," IEEE Commun. Surveys \& Tutorials, vol. PP, no. 99, pp. 1-1, 2015.

[142] R. Becker, R. Caceres, K. Hanson, J. Loh, S. Urbanek, A. Varshavsky, and C. Volinsky, "A tale of one city: Using cellular network data for urban planning," IEEE Pervasive Comput., vol. 10, no. 4, pp. 18-26, Apr. 2011

[143] H. T. Marques-Neto, F. H. Z. Xavier, W. Z. Xavier, C. H. S. Malab, A. Ziviani, L. M. Silveira, and J. M. Almeida, "Understanding human mobility and workload dynamics due to different large-scale events using mobile phone data," Journal of Netw. and Systems Management, vol. 26, no. 4, pp. 1079-1100, Oct 2018.

[144] R. Keralapura, A. Nucci, Z.-L. Zhang, and L. Gao, "Profiling users in a $3 \mathrm{~g}$ network using hourglass co-clustering," in Proc. ACM MobiCom, 2010, pp. 341-352.

[145] A. Vaccari, L. Liu, A. Biderman, C. Ratti, F. Pereira, J. Oliveirinha, and A. Gerber, "A holistic framework for the study of urban traces and the profiling of urban processes and dynamics," in Proc. IEEE ITSC, Oct 2009, pp. 1-6.

[146] E. Mucelli Rezende Oliveira, A. Carneiro Viana, K. P. Naveen, and C. Sarraute, "Mobile Data Traffic Modeling: Revealing Temporal Facets," Comput. Netw., vol. 112, pp. 176-193, Jan. 2017.

[147] A. Mehrotra, A. Nguyen, J. Blumenstock, and V. Mohan, "Differences in phone use between men and women: Quantitative evidence from rwanda," in Proc. ACM ICTD, 2012, pp. 297-306.

[148] I. Trestian, S. Ranjan, A. Kuzmanovic, and A. Nucci, "Measuring serendipity: Connecting people, locations and interests in a mobile $3 \mathrm{~g}$ network," in Proc. ACM SIGCOMM, 2009, pp. 267-279.

[149] U. Paul, A. P. Subramanian, M. M. Buddhikot, and S. R. Das, "Understanding traffic dynamics in cellular data networks," in Proc. IEEE INFOCOM, Apr. 2011, pp. 882-890.

[150] H. H. Jo, M. Karsai, J. Karikoski, and K. Kaski, "Spatiotemporal correlations of handset-based service usages," EPJ Data Science, vol. 1, pp. 1-18, 2012.

[151] G. Chen, S. Hoteit, A. C. Viana, M. Fiore, and C. Sarraute, "The spatiotemporal interplay of regularity and randomness in cellular data traffic," in Proc. IEEE LCN, Oct 2017, pp. 187-190.

[152] R. Ganti, F. Ye, and H. Lei, "Mobile crowdsensing: Current state and future challenges," IEEE ComMag, vol. 49, no. 11, pp. 32-39, Nov. 2011. 
[153] G. Ranjan, H. Zang, Z.-L. Zhang, and J. Bolot, "Are call detail records biased for sampling human mobility?" ACM SIGMOBILE, vol. 16, no. 3, p. 33, Dec. 2012.

[154] T. Tsai and H. Chan, "Nccu trace: social-network-aware mobility trace," IEEE ComMag, vol. 53, no. 10, pp. 144-149, Oct. 2015.

[155] K. Thilakarathna, A. C. Viana, A. Seneviratne, and H. Petander, "Design and analysis of an efficient friend-to-friend content dissemination system," IEEE Trans. on Mobile Comput., vol. 16, no. 3, pp. 702-715, Mar. 2017.

[156] L. R. Goldberg, "A broad-bandwidth, public domain, personality inventory measuring the lower-level facets of several five-factor models," Personality Psychology in Europe, Tilburg University Press, vol. 1, no. 7, pp. 7-28, 1999 .

[157] M. C. Gonzalez, C. Hidalgo, and A.-L. Barabasi, "Understanding individual human mobility patterns," Nature, vol. 453, pp. 779-82, 07 2008.

[158] A. G. Miklas, K. K. Gollu, K. K. W. Chan, S. Saroiu, K. P. Gummadi, and E. De Lara, "Exploiting social interactions in mobile systems," in Proc. Springer UbiComp, 2007, pp. 409-428.

[159] M. V. Barbera, A. C. Viana, M. D. de Amorim, and J. Stefa, "Data offloading in social mobile networks through vip delegation," Ad Hoc Netw., vol. 19, pp. $92-110,2014$

[160] E. G. C. . and A. M. Rinaldi., "Big data visualization tools: A survey - the new paradigms, methodologies and tools for large data sets visualization," in Proc. Int. Conf. on Data Science, Technol. and Appl., 2017, pp. 296-305.

[161] P. Andruszkiewicz and H. Rybinski, "Data acquisition and information extraction for scientific knowledge base building," in Proc. IEEE ICSC, Jan 2018, pp. 256-259.

[162] A. Khan, J. A. Doucette, R. Cohen, and D. J. Lizotte, "Integrating machine learning into a medical decision support system to address the problem of missing patient data," in Proc. IEEE ICMLA, vol. 1, Dec 2012, pp. 454-457.

[163] A. M. Pradhan and A. S. Varde, "Ontology based meta knowledge extraction with semantic web tools for ubiquitous computing," in Proc. IEEE UEMCON, Oct 2016, pp. 1-6.

[164] F. Kudo, T. Akitomi, and N. Moriwaki, "An artificial intelligence computer system for analysis of social-infrastructure data," in Proc. IEEE CBI, vol. 1, Jul. 2015, pp. 85-89.

[165] A. Di Luzio, A. Mei, and J. Stefa, "Consensus robustness and transaction de-anonymization in the ripple currency exchange system," in Proc. IEEE ICDCS, Jun. 2017, pp. 140-150.

[166] A. Heifetz, V. Mugunthan, and L. Kagal, "Shade: A differentiallyprivate wrapper for enterprise big data," in Proc. IEEE BigData, Dec 2017, pp. 1033-1042.

[167] S. H. Begum and F. Nausheen, "A comparative analysis of differential privacy vs other privacy mechanisms for big data," in Proc. IEEE ICISC, Jan 2018, pp. 512-516.

[168] T. Karle and D. Vora, "Privacy preservation in big data using anonymization techniques," in Proc. Int. Conf. on Data Management, Analytics and Innovation, Feb 2017, pp. 340-343.

[169] Y. Canbay, Y. Vural, and S. Sagiroglu, "Privacy preserving big data publishing," in Proc. IEEE IBIGDELFT, Dec 2018, pp. 24-29.

[170] J. Andrew, J. Karthikeyan, and J. Jebastin, "Privacy preserving big data publication on cloud using mondrian anonymization techniques and deep neural networks," in Proc. IEEE ICACCS, Mar. 2019, pp. $722-727$.

[171] B. K. Samanthula, "Privacy-preserving outsourced collaborative frequent itemset mining in the cloud," in Proc. IEEE BigData, Dec 2017, pp. 4827-4829.

[172] S. Wang, R. Sinnott, and S. Nepal, "Privacy-protected place of activity mining on big location data," in Proc. IEEE BigData, Dec 2017, pp. 1101-1108.

[173] C. K. Leung, C. S. H. Hoi, A. G. M. Pazdor, B. H. Wodi, and A. Cuzzocrea, "Privacy-preserving frequent pattern mining from big uncertain data," in Proc. IEEE BigData, Dec 2018, pp. 5101-5110.

[174] A. Cuzzocrea and C. Mastroianni, "A general overview of privacypreserving big data management and analytics models, methods and techniques in specific domains: Static and dynamic distributed environments," in Proc. IEEE BigData, Dec 2018, pp. 5093-5100.

[175] A. Cuzzocrea and E. Damiani, "Making the pedigree to your big data repository: Innovative methods, solutions, and algorithms for supporting big data privacy in distributed settings via data-driven paradigms," in Proc. IEEE COMPSAC, vol. 2, Jul 2019, pp. 508-516.

[176] A. Pereira Ferreira and R. Sinnott, "A performance evaluation of containers running on managed kubernetes services," in Proc. IEEE CloudCom, 2019, pp. 199-208.
[177] W. Yu, H. Xu, H. Zhang, D. Griffith, and N. Golmie, "Ultra-dense networks: Survey of state of the art and future directions," in Proc. IEEE ICCCN, Aug 2016, pp. 1-10.

[178] V. S. Subrahmanian and S. Kumar, "Predicting human behavior: The next frontiers," Science, vol. 355, no. 6324, pp. 489-489, 2017.

[179] E. M. R. Oliveira and A. C. Viana, "From routine to network deployment for data offloading in metropolitan areas," in Proc. IEEE SECON, Jun. 2014, pp. 126-134

[180] R. Ladjel, N. Anciaux, P. Pucheral, and G. Scerri, "Trustworthy distributed computations on personal data using trusted execution environments," in IEEE TrustCom/BigDataSE, 2019, pp. 381-388.

[181] Upguard, "The rnc files: Inside the largest us voter data leak," https://www.upguard.com/breaches/the-rnc-files, 2017, accessed: 2020$11-05$.

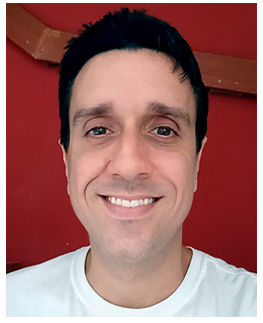

Rafael Lima Costa is a Ph.D. candidate at the Institute Polytechnique de Paris and Federal University of Bahia, Brazil. He received a M.Sc. degree in Computing \& Systems (2009) and a B.Sc. in Computer Science (2006), both from the University of Salvador, Brazil. He worked on several projects for the Brazilian National Education and Research Network (RNP) and as an IT consultant for private and public institutions. His research interests include future Internet architectures, human-aware networking solutions, and network performance analysis.

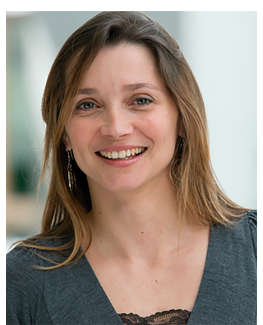

Aline Carneiro Viana is a permanent Reseach Director at Inria, she got her habilitation degree in 2011 and her PhD in Computer Science in 2005, both from UPMC - Sorbonne Universités, France, and a postdoctoral position at IRISA/Inria Rennes. Her research interests include solutions for tactful networking, smart cities, mobile and self-organizing networks. She is a recipient of the French Scientific Excellence award since 2014, and a N2Women award in 2016. Aline is also a member of IEEE, $\mathrm{ACM}$, Springer, and Elsevier.

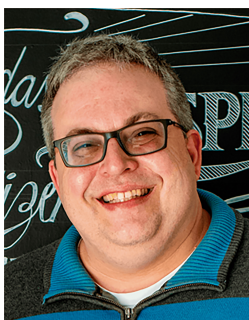

Artur Ziviani (S'99, M'04, SM'11) is a Senior Researcher at National Laboratory for Scientific Computing (LNCC), Brazil. In 2003, he received a $\mathrm{Ph} . \mathrm{D}$. in Computer Science from UPMC - Sorbonne Universités, Paris, France. His current research interests include network modeling and analysis; network science; and interdisciplinary data science with a networking approach. He is a Member of SBC (the Brazilian Computer Society), an Affiliated Member of the Brazilian Academy of Sciences (2013-2017), and a Senior Member of both IEEE and ACM.

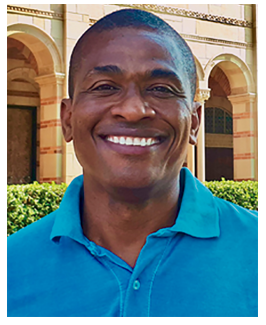

Leobino Nascimento Sampaio is an Associate Professor of Computer Science at the Federal University of Bahia (UFBA). He holds a Ph.D. degree in Computer Science from the Federal University of Pernambuco (UFPE), awarded in 2011. Currently, he is a visiting researcher at Computer Science Department of University of California, Los Angeles (UCLA) in United States and a member of SBC (the Brazilian Computer Society), ACM, and IEEE. His research interests include future Internet architectures and network performance evaluation. 